\title{
$N, N$ 'bis(2-(benzylthio)ethyl)malonamides: Synthesis, Electronic and Steric effects in Silver(I) Extraction and Silver(I) Binding Studies
}

\author{
Abiodun D. Aderibigbe*1,2 and Andrew J. Clark ${ }^{1}$ \\ ${ }^{1}$ Department of Chemistry, University of Warwick, Coventry CV4 7AL. \\ ${ }^{2}$ Department of Chemistry, The Federal University of Technology Akure, P.M.B. 704 Akure, Ondo state, \\ Nigeria.
}

\begin{abstract}
Unsubstituted and gem-diethyl substituted malonamide derivatives, bearing benzylthio arms were prepared in moderate to high yields at room temperature. It was observed that electronics effects at the 4-aryl position in the malonamide derivatives had a significant effect on the selectivity, but little effect on silver(I) extraction efficiency measured by liquid-liquid extraction experiments (with the 4-methoxy analogue proving the most selective). Increased steric hindrance near the sulfur donor had a small negative effect on silver(I) extraction efficiency, while hindrance at the methylene centre reduces selectivity. One of the malonamide derivatives prepared was found to bind to silver(I) in a 1:1 fashion suggesting a tetrahedral coordination type.
\end{abstract}

Keywords: Malonamide derivatives, selective silver(I) extraction, electronics, sterics, Job's and mole ratio plots.

\section{Introduction}

As the world population increases, the demand for products like phones, laptops, batteries, jewellery etc., containing precious metals like silver is certain to increase as well.[1-3] Unfortunately, silver just like other metals are non-renewable resources. Therefore, in order to meet the increasing demand, the supply of silver must be guaranteed. Two major options that have been explored to guarantee the supply of silver and indeed other metals is the search for new primary sources[4], (new metal ore deposits) and the secondary sources or urban mining, in other words, recycling of metals[5]. The success of these options rests on the development of an efficient, metal selective and cost-effective $\mathrm{Ag}^{+}$recovery process.

$N, N^{\prime}$-malonamide derivatives (MADs) such as 1a (Figure 1) have attracted attention due to their versatility for metal recovery in separation science, having demonstrated outstanding efficiency and selectivity in the recovery of highly valuable metals including lanthanides, actinides and precious metals[6-9]. The presence of two acyl groups and an active methylene centre allows the modification of the structural features of this ligand towards accessing derivatives with selective metal recovery and desired solubility properties.[9] Addition of specific donors has been shown to improve the efficiency 
and selectivity of the MADs for metal recovery. For example, Daubinet and Kaye[9], prepared a range of MADs (1a-d) (Figure 1) with different donor groups and varying degrees of lipohilicities via a microwave-assisted method and observed that the malonamide derivative 1a bearing a benzylthio arm demonstrated the highest efficiency $(97 \%)$ and selectivity of $\mathrm{Ag}^{+}$recovery from a solution also containing $\mathrm{Cu}^{2+}$ and $\mathrm{Pb}^{2+}$.

As part of an ongoing program exploring the development of in situ technologies for recovery of precious metals, from waste repositories (including metallurgical slag heaps, mine tailings and possibly landfills), we queried the possibility of accessing a $\mathrm{Ag}^{+}$-selective ligand bearing a vinyl handle which can be tethered by polymerization to a magnetic nanoparticle. The malonamide derivative 1e, (Figure 1) was chosen as our initial target after we had judged that it could be easily prepared and that it has high stability in low $\mathrm{pH}$ environments. Unfortunately, previously reported conditions for accessing the related malonamide derivative ligand 1a involve high temperatures which we feared could occasion the polymerization of the important vinyl group. Therefore, initial study focused on the development of a room temperature method to accessing derivatives of 1a; 1e-i (Figure 2). Subsequently the effect of electronics (using 1a and 1e-h) and sterics (using 1a, 1i and 3a) (Figure 2) on $\mathrm{Ag}^{+}$extraction was investigated to understand how modification affects the selectivity and efficiency of the ligand. Finally, the binding stoichiometry of the $\mathrm{Ag}^{+}-\mathbf{1 a}$ complex was thoroughly investigated to gain an insight into the $\mathrm{Ag}^{+}$binding nature of the ligand $\mathbf{1 a}$.<smiles>O=C(CC(=O)NCCSCc1ccccc1)NCCSCc1ccccc1</smiles><smiles>COc1ccccc1NC(=O)CC(=O)Nc1ccccc1CNC(=O)CC(=O)Nc1ccccc1C(C)(C)C</smiles><smiles>C=Cc1ccc(CSCCNC(=O)CC(=O)NCCSCc2ccc(C=C)cc2)cc1</smiles>

Figure 1. $N, N^{\prime}$-malonamide derivatives 1a-e. 
<smiles>[X]c1ccc(CSC([R])([R])CNC(=O)CC([R])([R])SCc2ccc([X])cc2)cc1</smiles>

1a; $\mathrm{R}=\mathrm{H}, \mathrm{X}=\mathrm{H}$

1e; $\mathrm{R}=\mathrm{H}, \mathrm{X}=\mathrm{CH}_{2}=\mathrm{CH}$

1f; $\mathrm{R}=\mathrm{H}, \mathrm{X}=\mathrm{MeO}$

1g; $\mathrm{R}=\mathrm{H}, \mathrm{X}=\mathrm{F}$

1h; $\mathrm{R}=\mathrm{H}, \mathrm{X}=\mathrm{NO}_{2}$

1i; $\mathrm{R}=\mathrm{CH}_{3}, \mathrm{X}=\mathrm{H}$<smiles>[X]c1ccc(CSCCNC(=O)C(CC)(CC)C(=O)NCCSCc2ccc([X])cc2)cc1</smiles>

3a; $\mathrm{X}=\mathrm{H}$

3b; $X=\mathrm{CH}_{2}=\mathrm{CH}_{2}$

3c; $X=\mathrm{MeO}$

3d; $\mathrm{X}=\mathrm{F}$

3e; $\mathrm{X}=\mathrm{NO}_{2}$

Figure 2. $N, N^{\prime}$-malonamide derivatives synthesized in this work

\section{Material and methods}

\section{Materials and instrumentation}

All reagents were purchased from commercial sources and used as received. ${ }^{1} \mathrm{H}$ and ${ }^{13} \mathrm{C}$ NMR were recorded at room temperature on $\mathrm{Bruker}^{\odot}$ Advance spectrometers and all chemical shift values were referenced to an internal standard of tetramethylsilane $(\delta=0.0 \mathrm{ppm})$. Fourier transform infra-red (FTIR) spectra were recorded on Bruker ${ }^{\odot}$ Alpha Platinum-Attenuated Total Reflectance IR spectrometer. All accurate mass spectra were run on a Bruker ${ }^{\odot}$ MaXis mass spectrometer. Metal concentrations were measured by means of a PerkinElmer 5300DV Inductively Coupled Plasma Optical Emission spectrophotometer (ICP-OES).

\section{Methods}

\section{Syntheses}

\section{S-benzylcysteamines $(2 a-e)$.}

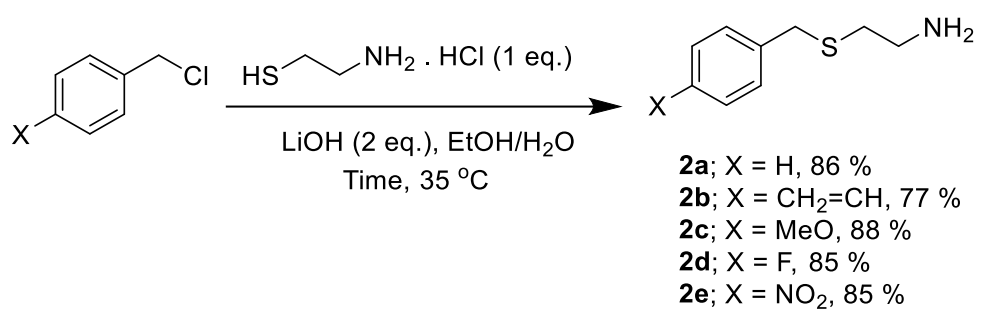

Scheme 1. Syntheses of $S$-benzylcysteamines 2a-e[10]

The benzylcysteamines 2a-e were synthesized following the method reported by Ghosh and Tochtrop[10]. Briefly, to a stirred water/ethanol (1:3) solution of LiOH (2 eq.) and 2-aminoethanethiol hydrochloride ( 1 eq.) was added dropwise for $5 \mathrm{~min}$, the appropriate benzyl chlorides ( 1 eq.) and the reaction was left to stir at $35{ }^{\circ} \mathrm{C}$ for $20 \mathrm{~min}$ (for $\mathbf{2 a}$ ) or $40 \mathrm{~min}$ (for $\mathbf{2 b - e )}$ ) after which the solvent was removed in vacuo (Scheme 1). The crude mixture was extracted with dichloromethane (DCM) after it 
had been solubilized with water. The DCM extract was then dried with anhydrous $\mathrm{Na}_{2} \mathrm{SO}_{4}$ or $\mathrm{MgSO}_{4}$, filtered and concentrated in vacuo. All amines except for $\mathbf{2 d}$ (the fluoro analogue) were obtained as oils needing no purification. The amine $\mathbf{2 d}$ was subsequently purified by column chromatography using a mobile phase gradient of $100 \%$ EtOAc to $50 \%$ v/v EtOAc/MeOH.

2-(Benzylthio)ethan-1-amine (2a).[10] Viscous colourless oil. Yield: $5.76 \mathrm{~g}(86 \%), \mathrm{v}\left(\mathrm{cm}^{-1}\right)$ 3363, 3279 (N-H stretch), 1600 (N-H bend), ${ }^{1} \mathrm{H}$ NMR (400 MHz, $\left.\mathrm{CDCl}_{3}\right) \delta 7.39-7.18$ (m, 5H, $\mathrm{Ar} \underline{H}$ ), 3.70 (s, 2H, $\left.\mathrm{PhC} \underline{H}_{2} \mathrm{~S}\right), 2.81$ (t, $\left.J=6.5 \mathrm{~Hz}, 2 \mathrm{H}, \mathrm{SCH}_{2} \underline{\mathrm{C}}_{2}\right), 2.51\left(\mathrm{t}, J=6.5 \mathrm{~Hz}, 2 \mathrm{H}, \mathrm{SC} \underline{H}_{2} \mathrm{CH}_{2}\right), 1.29$ (s, $2 \mathrm{H}$, $\mathrm{CH}_{2} \mathrm{CH}_{2} \mathrm{~N}_{2}$ ), ${ }^{13} \mathrm{C}$ NMR (100 MHz, $\left.\mathrm{CDCl}_{3}\right)$ 138.5, 128.9, 128.6 and 127.1 (ArC), $40.9\left(\mathrm{CH}_{2} \underline{C H}_{2} \mathrm{NH}_{2}\right)$, $36.0\left(\mathrm{PhCH}_{2} \mathrm{~S}\right), 35.7\left(\mathrm{SCH}_{2} \mathrm{CH}_{2}\right) ; \mathrm{m} / z(\mathrm{ESI}): 168[\mathrm{M}+\mathrm{H}]^{+}$.

2-((4-Vinylbenzyl)thio)ethan-1-amine (2b). Viscous yellow oil. Yield: $5.87 \mathrm{~g}(77 \%),, \mathrm{v}\left(\mathrm{cm}^{-1}\right) 3365$, (N-H stretch), 1627, 1567 (N-H bend), ${ }^{1} \mathrm{H}$ NMR (300 MHz, $\left.\mathrm{CDCl}_{3}\right) \delta 7.36$ (d, $\left.J=8.0 \mathrm{~Hz}, 2 \mathrm{H}, \mathrm{ArH}\right)$, $7.27(\mathrm{~d}, J=8.0 \mathrm{~Hz}, 2 \mathrm{H}, \mathrm{ArH}), 6.70$ (dd, $\left.J=17.5,11.0 \mathrm{~Hz}, 1 \mathrm{H}, \mathrm{CH}_{2} \mathrm{C} \underline{H P h}\right), 5.73$ (d, $J=17.5 \mathrm{~Hz}, 1 \mathrm{H}$, $\left.\underline{\mathrm{C}}_{\mathrm{a}} \mathrm{H}_{\mathrm{b}} \mathrm{CHPh}\right), 5.23\left(\mathrm{~d}, J=11.0 \mathrm{~Hz}, 1 \mathrm{H}, \mathrm{CH}_{\mathrm{a}} \underline{H}_{\mathrm{b}} \mathrm{CHPh}\right), 3.69$ (s, $\left.2 \mathrm{H}, \mathrm{PhC} \underline{H}_{2} \mathrm{~S}\right), 2.82(\mathrm{t}, J=6.0 \mathrm{~Hz}, 2 \mathrm{H}$, $\mathrm{CH}_{2} \underline{\mathrm{C}}_{2} \mathrm{NH}_{2}$ ), 2.51 (t, $J=6.0 \mathrm{~Hz}, 2 \mathrm{H}, \mathrm{SC} \underline{H}_{2} \mathrm{CH}_{2}$ ), 1.29 (s, $2 \mathrm{H}, \mathrm{CH}_{2} \mathrm{CH}_{2} \mathrm{~N}_{2}$ ), ${ }^{13} \mathrm{C}$ NMR (75 MHz, $\left.\mathrm{CDCl}_{3}\right) \delta 138.1$ and $136.5(\mathrm{ArC}), 136.4\left(\mathrm{CH}_{2} \underline{C} \mathrm{HPh}\right), 129.1$ and $126.5(\mathrm{ArC}), 113.9\left(\underline{C}_{2} \mathrm{CHPh}\right), 41.0$

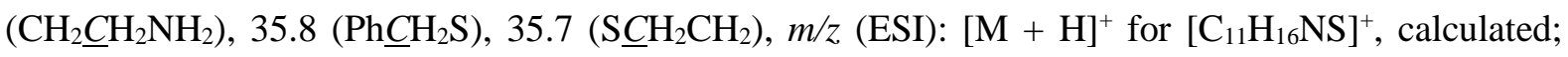
194.1003, found 194.0998.

2-((4-Methoxybenzyl)thio)ethan-1-amine (2c).[10] Viscous colourless oil. Yield: $4.37 \mathrm{~g} \mathrm{(88 \% ),}\left(\mathrm{cm}^{-}\right.$ $\left.{ }^{1}\right): 3261$ (N-H stretch), 1663 (N-H bend), ${ }^{1} \mathrm{H}$ NMR (400 MHz, $\left.\mathrm{CDCl}_{3}\right) \delta 7.23$ (d, J=8.5 Hz, 2H and $6.84(\mathrm{~d}, J=8.5 \mathrm{~Hz}, 2 \mathrm{H})(\mathrm{ArH}), 3.79\left(\mathrm{~s}, 3 \mathrm{H}, \mathrm{C} \underline{H}_{3} \mathrm{OPh}\right), 3.67$ (s, $\left.2 \mathrm{H}, \mathrm{PhC} \underline{H}_{2} \mathrm{~S}\right), 2.85$ (t, $J=6.5 \mathrm{~Hz}, 2 \mathrm{H}$, $\mathrm{CH}_{2} \underline{\mathrm{C}}_{2} \mathrm{NH}_{2}$ ), 2.71 (s, $2 \mathrm{H}, \mathrm{CH}_{2} \mathrm{CH}_{2} \mathrm{~N}_{2}$ ), 2.55 (t, $\left.J=6.5 \mathrm{~Hz}, 2 \mathrm{H}, \mathrm{SC}_{2} \mathrm{CH}_{2}\right) .{ }^{13} \mathrm{C} \mathrm{NMR}(100 \mathrm{MHz}$, $\left.\mathrm{CDCl}_{3}\right) \delta 159.0,130.2,130.0$ and $114.1(\mathrm{ArC}), 55.4\left(\underline{\mathrm{C}} \mathrm{H}_{3} \mathrm{OPh}\right), 40.5\left(\mathrm{CH}_{2} \underline{\mathrm{C}} \mathrm{H}_{2} \mathrm{NH}_{2}\right), 35.4\left(\mathrm{Ph}_{\underline{C}} \mathrm{H}_{2} \mathrm{~S}\right)$, $34.5\left(\mathrm{~S}_{\mathrm{CH}} \mathrm{CH}_{2}\right), m / z(\mathrm{ESI}): 198[\mathrm{M}+\mathrm{H}]^{+}$.

2-((4-Fluorobenzyl)thio)ethan-1-amine (2d).[10] Viscous colourless oil. Yield: $4.27 \mathrm{~g}$ (85\%), v(cm$\left.{ }^{1}\right): 3365$ (N-H stretch), 1599 (N-H bend), ${ }^{1} \mathrm{H}$ NMR $\left(400 \mathrm{MHz}, \mathrm{CDCl}_{3}\right) \delta 7.35-7.23\left(\mathrm{dd}, 2 \mathrm{H}, J_{\mathrm{HCCH}}=9\right.$ $\left.\mathrm{Hz}, J_{\mathrm{HCCCH}}=3 \mathrm{~Hz}, \mathrm{ArH}\right), 7.06-6.96\left(\mathrm{t}, 2 \mathrm{H}, J_{\mathrm{HCCF}}=15 \mathrm{~Hz}, \mathrm{ArH}\right), 3.68\left(\mathrm{~s}, 2 \mathrm{H}, \mathrm{PhC} \underline{H}_{2} \mathrm{~S}\right), 2.82(\mathrm{t}, J=6.5$ $\left.\mathrm{Hz}, 2 \mathrm{H}, \mathrm{CH}_{2} \mathrm{C}_{2} \mathrm{NH}_{2}\right), 2.51$ (t, $\left.J=6.5 \mathrm{~Hz}, 2 \mathrm{H}, \mathrm{SC}_{2} \mathrm{CH}_{2}\right), 1.33$ (s, 2H, $\left.\mathrm{CH}_{2} \mathrm{CH}_{2} \mathrm{~N}_{2}{ }_{2}\right),{ }^{13} \mathrm{C}$ NMR (100 $\left.\mathrm{MHz}, \mathrm{CDCl}_{3}\right) \delta 162\left(\mathrm{~d}, J_{\mathrm{CF}}=244 \mathrm{~Hz}\right), 134.2\left(\mathrm{~d}, J_{\mathrm{CCCF}}=3 \mathrm{~Hz}\right), 130.4\left(\mathrm{~d}, J_{\mathrm{CCF}}=8 \mathrm{~Hz}\right)$ and $115.4(\mathrm{~d}$, $\left.J_{\mathrm{CCF}}=21 \mathrm{~Hz}\right)(\mathrm{ArC}), 40.9\left(\mathrm{CH}_{2} \underline{\mathrm{CH}} \mathrm{H}_{2} \mathrm{NH}_{2}\right), 35.6\left(\mathrm{Ph} \underline{\mathrm{CH}} \mathrm{H}_{2} \mathrm{~S}\right), 35.2\left(\mathrm{~S}_{\mathrm{CH}} \mathrm{CH}_{2}\right), \mathrm{m} / z(\mathrm{ESI}): 186[\mathrm{M}+\mathrm{H}]^{+}$.

2-((4-Nitrobenzyl)thio)ethan-1-amine (2e).[10] Yellow oil. Yield: $3.23 \mathrm{~g}(85 \%), \mathrm{v}\left(\mathrm{cm}^{-1}\right): 3365(\mathrm{~N}-\mathrm{H}$ stretch), 1599 (N-H bend), 1367 (N-O stretch), ${ }^{1} \mathrm{H}$ NMR (300 MHz, CDCl $) \delta 8.18$ (d, J=8.5 Hz, 2H, $\mathrm{ArH}$ ), 7.50 (d, J=8.5 Hz, 2H, ArH), 3.79 (s, $2 \mathrm{H}, \mathrm{PhC}_{2} \mathrm{~S}$ ), 2.85 (t, $J=6.5 \mathrm{~Hz}, 2 \mathrm{H}, \mathrm{CH}_{2} \mathrm{C}_{2} \mathrm{NH}_{2}$ ), 2.53 (t, $\left.J=6.5 \mathrm{~Hz}, 2 \mathrm{H}, \mathrm{SC}_{2} \mathrm{CH}_{2}\right), 1.38\left(\mathrm{~s}, 2 \mathrm{H}, \mathrm{CH}_{2} \mathrm{CH}_{2} \mathrm{~N}_{2}\right),{ }^{13} \mathrm{C} \mathrm{NMR}\left(75 \mathrm{MHz}, \mathrm{CDCl}_{3}\right) \delta 147.1$ (ArC),

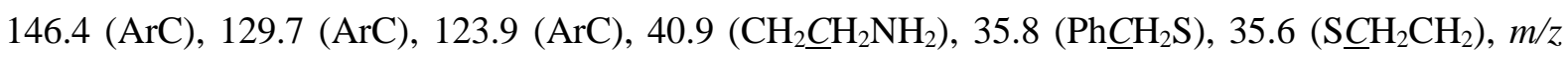
(ESI): $213[\mathrm{M}+\mathrm{H}]^{+}$. 


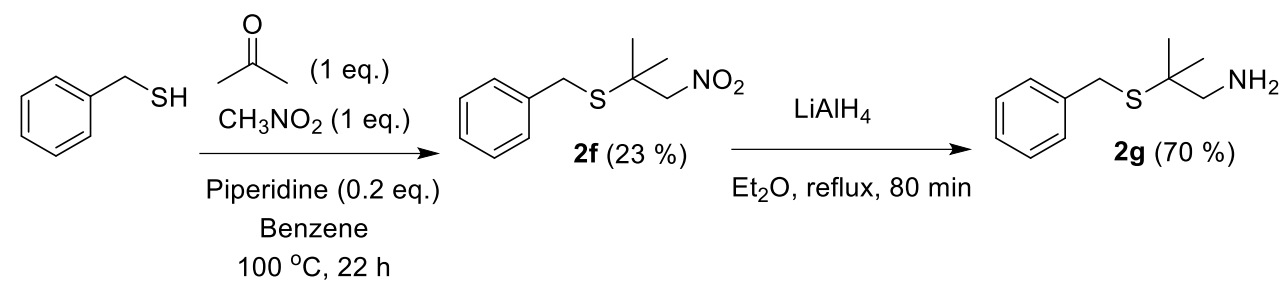

Scheme 2. Synthesis of the gem-dimethyl substituted S-benzylcysteamine $\mathbf{2 g [ 1 1 ]}$

Access to 2,2-dimethyl-2-benzylthioethyl amine $\mathbf{2 g}$ was achieved by following the method reported by Caroll and coworkers.[11] In the first step, a mixture of acetone (3.67 ml, $0.05 \mathrm{~mol})$, benzylmercaptan $(5.90 \mathrm{ml}, 0.05 \mathrm{~mol})$, nitromethane $(2.71 \mathrm{ml}, 0.05 \mathrm{~mol})$ and benzene $(18.75 \mathrm{ml})$ were heated at $100{ }^{\circ} \mathrm{C}$ inside a flask fitted with a Dean Stark apparatus filled with benzene (Scheme 2). After $22 \mathrm{~h}$, the crude mixture was left to cool and was washed with $2.0 \mathrm{M} \mathrm{HCl}$ and then with water. It was then dried with anhydrous $\mathrm{MgSO}_{4}$, concentrated in vacuo and purified by flash chromatography using $n$-hexane as eluent to yield 1-(benzylthio)-1,1-dimethylnitroethane $2 \mathbf{f}$ ( $2.60 \mathrm{~g}, 23 \%)$. In the second step, a dry ether solution of 1-(benzylthio)-1,1-dimethylnitroethane $\mathbf{2 f}$ ( $1 \mathrm{~g}, 4.4 \mathrm{mmol})$ was added to a cold ether solution of $\mathrm{LiAlH}_{4}(4 \mathrm{M}, 3.25 \mathrm{ml})$ inside a 2-necked flask. The mixture was left to stir for another $3 \mathrm{~min}$ after which it was transferred to a heating block and heated under reflux for $100 \mathrm{~min}$. The crude mixture was transferred to a $250 \mathrm{ml}$ flask and a stir bar added. Water and subsequently potassium sodium tartarate $(25 \mathrm{ml}, 20 \%)$ was added. The mixture was left to stir until all solids dissolved. The crude product was then extracted with ether $(20 \mathrm{~mL} \times 3)$ and purified by flash chromatography (16 - 50\% ethyl acetate: $n$-hexane, ethyl acetate and finally methanol) to give 2-(benzylthio)-2,2-dimethylmethylamine $\mathbf{2 f}$ as a yellow oil. Yield: $(0.60 \mathrm{~g}, 70 \%), \mathrm{v}\left(\mathrm{cm}^{-1}\right) 3376(\mathrm{NH}),{ }^{1} \mathrm{H}\left(300 \mathrm{MHz} \mathrm{CDCl}_{3}\right) 7.40-7.19$ (m, 5H, ArH), 3.68 (s, $\left.2 \mathrm{H}, \mathrm{PhC} \underline{H}_{2} \mathrm{~S}\right), 2.61$ (s, $\left.2 \mathrm{H}, \underline{\mathrm{C}}_{2} \mathrm{NH}_{2}\right), 1.44$ (s, $\left.2 \mathrm{H}, \mathrm{N} \underline{H}_{2}\right), 1.28$ (s, $\left.6 \mathrm{H}, \mathrm{SC}\left(\mathrm{C}_{3}\right)_{2}\right) ;{ }^{13} \mathrm{C}(75 \mathrm{MHz}$,

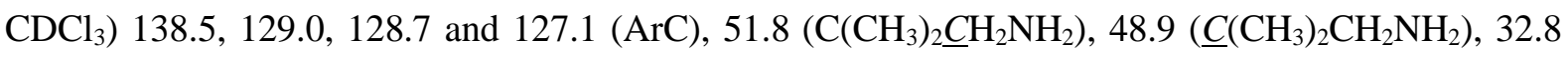
$\left(\mathrm{PhC} \mathrm{H}_{2} \mathrm{~S}\right), 26.6\left(\mathrm{C}\left(\underline{C H}_{3}\right)_{2} \mathrm{CH}_{2} \mathrm{NO}_{2}\right), \mathrm{m} / z(\mathrm{ESI}): 196[\mathrm{M}+\mathrm{H}]^{+}$.

\section{$N, N^{\prime}$-bis(2-benzylthio)ethyl)malonamides (1a and 1e-i)}

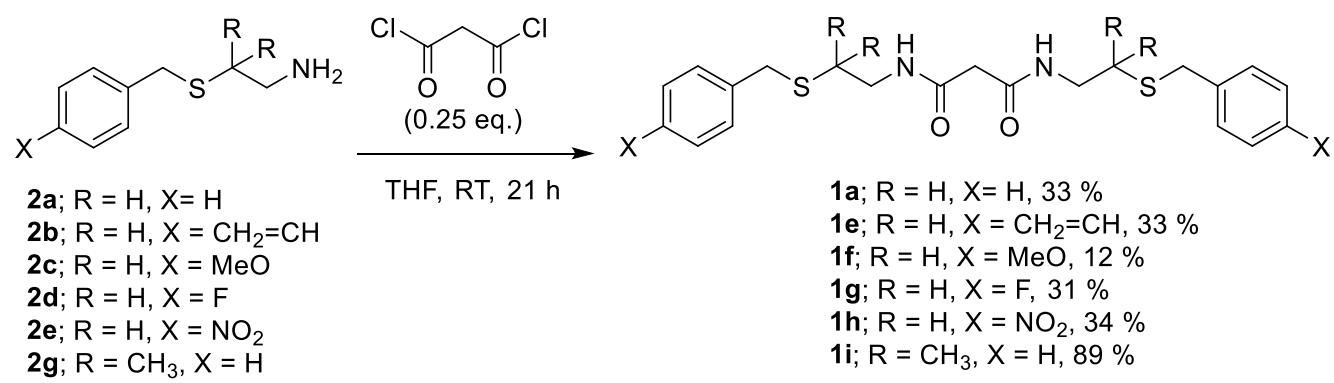

Scheme 3. Syntheses of the unsubstituted malonamide derivatives 1a and 1e-i 
To a stirred solution of the amine ( $1 \mathrm{eq})$ in dry THF, malonyl chloride $(0.25 \mathrm{eq})$ solution in dry THF was added dropwise for $90 \mathrm{~min}$. The reaction mixture was left to stir overnight (Scheme 3) and the THF solvent was removed in vacuo after which water was added and the crude product was extracted with EtOAc. The crude product was washed successively with $\mathrm{HCl}(2 \mathrm{M}), \mathrm{NaHCO}_{3}$, water and finally brine. It was then dried over $\mathrm{Na}_{2} \mathrm{SO}_{4}$ and concentrated in vacuo and purified by flash chromatography (20\% EtOAc/Hexane to $100 \%$ EtOAc) to give the malonamide derivatives 1a and 1e-i.

N,N'bis(2-(benzylthio)ethyl)malonamide (1a)[9] Yellow powder. Yield: $0.21 \mathrm{~g}$ (33 \%), m.p.: 108 $112{ }^{\circ} \mathrm{C}$ (lit.,[9]: $105-106{ }^{\circ} \mathrm{C}$ ), , $\mathrm{v}\left(\mathrm{cm}^{-1}\right): 3296$ (amide $\mathrm{N}-\mathrm{H}$ stretch), 1647 (amide $\mathrm{C}=\mathrm{O}$ bend), ${ }^{1} \mathrm{H}$ NMR (400 MHz, $\left.\mathrm{CDCl}_{3}\right) \delta 7.40-7.19$ (m, 10H, ArH), 7.05 (s, 2H, $\mathrm{CON}_{1} \mathrm{HCH}_{2}$ ), 3.71 (s, 4H, $\mathrm{PhC}_{2} \mathrm{~S}$ ), 3.39 (m, $4 \mathrm{H}, \mathrm{NHC}_{2} \mathrm{CH}_{2}$ ), 3.10 (s, $\left.2 \mathrm{H}, \mathrm{COC} \underline{H}_{2} \mathrm{CO}\right), 2.56\left(\mathrm{t}, J=6.5 \mathrm{~Hz}, 4 \mathrm{H}, \mathrm{CH}_{2} \underline{\mathrm{C}}_{2} \mathrm{~S}\right),{ }^{13} \mathrm{C} \mathrm{NMR}(100$ $\left.\mathrm{MHz}, \mathrm{CDCl}_{3}\right) \delta 167.3\left(\underline{\mathrm{COCH}}{ }_{2} \underline{\mathrm{CO}}\right), 138.2,129.0,128.8$ and $127.3(\mathrm{ArC}), 43.1\left(\mathrm{CO} \underline{\mathrm{C}} \mathrm{H}_{2} \mathrm{CO}\right), 38.5$ $\left(\mathrm{NH}_{\underline{C}} \mathrm{H}_{2} \mathrm{CH}_{2}\right), 36.0\left(\mathrm{Ph}_{\underline{C}} \mathrm{H}_{2} \mathrm{~S}\right), 30.9\left(\mathrm{CH}_{2} \underline{\mathrm{CH}} \mathrm{H}_{2} \mathrm{~S}\right), \mathrm{m} / z(\mathrm{ESI}): 425[\mathrm{M}+\mathrm{Na}]^{+}$.

N,N'-bis(2-((4-vinylbenzyl)thio)ethyl)malonamide (1e). Yellow powder. Yield: 0.09 g (33\%), m.p.: $151-156{ }^{\circ} \mathrm{C}, \mathrm{v}\left(\mathrm{cm}^{-1}\right): 3292$ (amide $\mathrm{N}-\mathrm{H}$ stretch), 1648 (amide $\mathrm{C}=\mathrm{O}$ bend), ${ }^{1} \mathrm{H}$ NMR $\left(400 \mathrm{MHz}, \mathrm{CDCl}_{3}\right.$ ) $\delta 7.36(\mathrm{~d}, J=7.5 \mathrm{~Hz}, 4 \mathrm{H})$ and $7.27(\mathrm{~d}, J=7.5 \mathrm{~Hz}, 4 \mathrm{H})(\mathrm{ArH}), 6.94\left(\mathrm{~s}, 2 \mathrm{H}, \mathrm{CON} \underline{H} \mathrm{CH}_{2}\right), 6.69(\mathrm{dd}, J=$ 17.5, 11.0 Hz, 2H, PhC $\left.\underline{H}_{\mathrm{c}} \mathrm{CH}_{\mathrm{a}} \mathrm{H}_{\mathrm{b}}\right), 5.73\left(\mathrm{~d}, J=17.5 \mathrm{~Hz}, 2 \mathrm{H}, \mathrm{PhCHC}_{\mathrm{H}} \mathrm{H}_{\mathrm{b}}\right), 5.24(\mathrm{~d}, J=11.0 \mathrm{~Hz}, 2 \mathrm{H}$, $\mathrm{PhCH}_{\mathrm{c}} \mathrm{CH}_{\mathrm{a}} \underline{\mathrm{H}}_{\mathrm{b}}$ ), 3.70 (s, $4 \mathrm{H}, \mathrm{PhC}_{2} \mathrm{~S}$ ), 3.40 (m, $\mathrm{CH}_{2} \underline{\mathrm{C}}_{2} \mathrm{NH}$ ), 3.11 (s, $2 \mathrm{H}, \mathrm{COC} \underline{H}_{2} \mathrm{CO}$ ), 2.56 (t, J = 6.5 $\left.\mathrm{Hz}, 4 \mathrm{H}, \mathrm{SC} \underline{H}_{2} \mathrm{CH}_{2}\right),{ }^{13} \mathrm{C}$ NMR (100 MHz, $\left.\mathrm{CDCl}_{3}\right) \delta 167.2\left(\underline{C O C H}_{2} \underline{\mathrm{CO}}\right), 137.7$ and $136.8(\mathrm{ArC}), 136.5$ $\left(\mathrm{CH}_{2} \underline{\mathrm{CHPh}}\right), 129.2$ and $\left.126.6(\mathrm{ArC}), 114.1(\mathrm{PhCH} \underline{\mathrm{CH}})_{2}\right), 43.1\left(\mathrm{CO} \underline{\mathrm{CH}} \mathrm{H}_{2} \mathrm{CO}\right), 38.4\left(\mathrm{CH}_{2} \underline{\mathrm{CH}} \mathrm{H}_{2} \mathrm{NH}\right), 35.7$ $\left(\underline{\mathrm{C}} \mathrm{H}_{2} \mathrm{SCH}_{2}\right), 30.8\left(\mathrm{~S}_{\mathrm{C}} \mathrm{H}_{2} \mathrm{CH}_{2}\right), \mathrm{m} / z(\mathrm{ESI}):[\mathrm{M}+\mathrm{Na}]^{+}$for $\left[\mathrm{C}_{25} \mathrm{H}_{27} \mathrm{~N}_{2} \mathrm{NaO}_{2} \mathrm{~S}_{2}\right]^{+}$calculated: 477.1646, found: 477.1643 .

N,N'-bis(2((4-methoxybenzyl)thio)ethyl)malonamide (1f). Yellow solid. Yield: 0.43 g (12\%), m.p.: $146-149^{\circ} \mathrm{C},, v\left(\mathrm{~cm}^{-1}\right): 3337(\mathrm{NH}), 1650$ (amide $\mathrm{C}=\mathrm{O}$ bend), $1240\left(\mathrm{C}-\mathrm{O}\right.$; aromatic ether), ${ }^{1} \mathrm{H}$ NMR (300 MHz, $\left.\mathrm{CDCl}_{3}\right) \delta 7.24$ (d, J=8.5 Hz, 4H, ArH), 6.97 (s, 2H, $\mathrm{CH}_{2} \mathrm{CH}_{2} \mathrm{~N} \underline{H}$ ), 6.85 (d, J = 8.5 Hz, 4H, $\mathrm{ArH}$ ), 3.79 (s, $6 \mathrm{H}, \underline{\mathrm{C}}_{3} \mathrm{OPh}$ ), 3.67 (s, 4H, PhC $\underline{H}_{2} \mathrm{~S}$ ), 3.40 (m, 4H, $\mathrm{CH}_{2} \mathrm{C} \underline{H}_{2} \mathrm{NH}$ ), 3.11 (s, $2 \mathrm{H}, \mathrm{COC} \underline{H}_{2} \mathrm{CO}$ ), $2.55\left(\mathrm{t}, J=6.5 \mathrm{~Hz}, 4 \mathrm{H}, \mathrm{SC} \underline{H}_{2} \mathrm{CH}_{2}\right),{ }^{13} \mathrm{C} \mathrm{NMR}\left(75 \mathrm{MHz}, \mathrm{CDCl}_{3}\right) \delta 167.2\left(\underline{C O C H}_{2} \mathrm{CO}\right), 158.6(\mathrm{ArC})$, 130.1 (ArC), 130.0 (ArC), $114.2(\mathrm{ArC}), 55.4\left(\underline{\mathrm{C}} \mathrm{H}_{3} \mathrm{O}\right), 43.1\left(\mathrm{CO}_{\underline{C}} \mathrm{H}_{2}\right), 38.5\left(\mathrm{CH}_{2} \underline{C H}_{2} \mathrm{NH}\right), 35.4$ $\left(\mathrm{Ph} \underline{\mathrm{C}} \mathrm{H}_{2} \mathrm{~S}\right), 30.8\left(\mathrm{~S}_{\mathrm{C}} \mathrm{H}_{2} \mathrm{CH}_{2}\right), \mathrm{m} / z(\mathrm{ESI}):[\mathrm{M}+\mathrm{Na}]^{+}$for $\left[\mathrm{C}_{23} \mathrm{H}_{30} \mathrm{~N}_{2} \mathrm{NaO}_{4} \mathrm{~S}_{2}\right]^{+}$calculated: 485.1545 , found: 485.1539.

N,N'-bis(2-((4-fluorobenzyl)thio)ethyl)malonamide (1g). Yellow powder. Yield: $0.55 \mathrm{~g}$ (31\%), m.p.: $121-123{ }^{\circ} \mathrm{C}, \mathrm{v}\left(\mathrm{cm}^{-1}\right): 3296$ (amide $\mathrm{N}-\mathrm{H}$ ), 1647 (amide $\mathrm{C}=\mathrm{O}$ bend), ${ }^{1} \mathrm{H}$ NMR $\left(400 \mathrm{MHz}, \mathrm{CDCl}_{3}\right) \delta$ $7.28\left(\mathrm{~d}, J_{\mathrm{HCCF}}=13.5 \mathrm{~Hz}, 4 \mathrm{H}, \mathrm{ArH}\right), 7.18\left(\mathrm{~s}, 2 \mathrm{H}, \mathrm{CH}_{2} \mathrm{~N} \underline{H C O}\right), 6.99(\mathrm{t}, J=8.5 \mathrm{~Hz}, 4 \mathrm{H}, \mathrm{ArH}), 3.69$ (s, $4 \mathrm{H}, \mathrm{PhC} \underline{H}_{2} \mathrm{~S}$ ), 3.41 (m, 4H, $\mathrm{CH}_{2} \underline{\mathrm{C}}_{2} \mathrm{NH}$ ), 3.15 (s, $2 \mathrm{H}, \mathrm{COC} \underline{H}_{2} \mathrm{CO}$ ), 2.55 (t, J=6.5 Hz, 4H, SC $\underline{H}_{2} \mathrm{CH}_{2}$ ), ${ }^{13} \mathrm{C}$ NMR $\left(100 \mathrm{MHz}, \mathrm{CDCl}_{3}\right) 167.3\left(\underline{C O C H}_{2} \mathrm{CO}\right), 162.1\left(J_{\mathrm{CF}}=242, \mathrm{FArC}\right), 133.8\left(J_{\mathrm{CCCCF}}=3 \mathrm{~Hz}, \mathrm{ArC}\right)$, 130.6 and $130.5\left(\mathrm{~d}, J_{\mathrm{CCCF}}=8 \mathrm{~Hz}\right), 115.7$ and $115.5\left(\mathrm{~d}, J_{\mathrm{CCF}}=21 \mathrm{~Hz}\right), 43.1\left(\mathrm{CO} \underline{C} \mathrm{H}_{2} \mathrm{CO}\right), 38.5$ 
$\left(\mathrm{CH}_{2} \underline{\mathrm{C}} \mathrm{H}_{2} \mathrm{NH}\right), 35.2\left(\mathrm{FPh} \underline{\mathrm{C}} \mathrm{H}_{2} \mathrm{~S}\right), 30.8\left(\mathrm{CH}_{2} \underline{\mathrm{C}} \mathrm{H}_{2} \mathrm{~S}\right), \mathrm{m} / z(\mathrm{ESI}):[\mathrm{M}+\mathrm{Na}]^{+}$for $\left[\mathrm{C}_{21} \mathrm{H}_{24} \mathrm{~F}_{2} \mathrm{~N}_{2} \mathrm{NaO}_{2} \mathrm{~S}_{2}\right]^{+}$ calculated: 461.1145 found: 461.1143 .

N,N'-bis(2-((4-nitrobenzyl)thio)ethyl)malonamide (1h). Yellow solid. Yield: 1.22 g (34\%), m.p.: 82 $85{ }^{\circ} \mathrm{C}, \mathrm{v}\left(\mathrm{cm}^{-1}\right): 3294$ (N-H stretch), 1647 (amide $\mathrm{C}=\mathrm{O}$ bend), 1544 and 1367 (N-O stretch), ${ }^{1} \mathrm{H}$ NMR $\left(300 \mathrm{MHz}, \mathrm{CDCl}_{3}\right) \delta 8.17(\mathrm{~d}, J=8.5 \mathrm{~Hz}, 4 \mathrm{H}, \mathrm{ArH}), 7.50$ (d, $\left.J=8.5 \mathrm{~Hz}, 4 \mathrm{H}, \mathrm{ArH}\right), 7.19$ (s, 2H, $\mathrm{CH}_{2} \mathrm{CH}_{2} \mathrm{~N} \underline{H}$ ), 3.80 (s, $\left.4 \mathrm{H}, \mathrm{PhCH}_{2} \mathrm{~S}\right), 3.43$ (m, 4H, $\mathrm{CH}_{2} \underline{C H}_{2} \mathrm{NH}$ ), 3.16 (s, $2 \mathrm{H}, \mathrm{COC} \underline{H}_{2} \mathrm{CO}$ ), 2.58 (t, $J=$ $\left.6.5 \mathrm{~Hz}, 4 \mathrm{H}, \mathrm{SC} \underline{H}_{2} \mathrm{CH}_{2}\right) .{ }^{13} \mathrm{C} \mathrm{NMR}\left(75 \mathrm{MHz}, \mathrm{CDCl}_{3}\right) \delta 167.3,\left(\underline{\mathrm{COCH}_{2}}\right), 147.2(\mathrm{ArC}), 145.9(\mathrm{ArC}), 129.9$ (ArC), 124.0 (ArC), $42.9\left(\mathrm{CO} \underline{C} \mathrm{H}_{2}\right), 38.4\left(\mathrm{CH}_{2} \underline{\mathrm{C}} \mathrm{H}_{2} \mathrm{NH}\right), 35.4\left(\mathrm{Ph}_{\underline{C}} \mathrm{H}_{2} \mathrm{~S}\right), 31.0\left(\mathrm{~S}_{\mathrm{CH}} \mathrm{CH}_{2}\right), \mathrm{m} / z(\mathrm{ESI})$ : $[\mathrm{M}+\mathrm{Na}]^{+}$for $\left[\mathrm{C}_{21} \mathrm{H}_{24} \mathrm{~N}_{4} \mathrm{NaO}_{6} \mathrm{~S}_{2}\right]^{+}$calculated: 515.1035, found: 515.1029.

N,N'bis(2-(benzylthio)-2-methylpropyl)malonamide (1i). Cream solid. Yield: $0.68 \mathrm{~g}$ (89\%), m.p.: 71 $-74{ }^{\circ} \mathrm{C},, v\left(\mathrm{~cm}^{-1}\right): 3260$ (amide $\mathrm{N}-\mathrm{H}$ stretch), 1663 (amide $\mathrm{C}=\mathrm{O}$ bend), ${ }^{1} \mathrm{H}$ NMR $\left(300 \mathrm{MHz}, \mathrm{CDCl}_{3}\right) \delta$ $7.40-7.20$ (m, 10H, ArH), 7.01 (s, 2H, $\mathrm{CH}_{2} \mathrm{~N} \underline{H C O}$ ), 3.71 (s, $\left.4 \mathrm{H}, \mathrm{PhCH}_{2} \mathrm{~S}\right), 3.32$ (d, J = 6.0 Hz, 4H, $\left.\mathrm{C}\left(\mathrm{CH}_{3}\right)_{2} \mathrm{C}_{2} \mathrm{NH}\right), 3.11$ (s, $\left.2 \mathrm{H}, \mathrm{COC} \underline{H}_{2} \mathrm{CO}\right), 1.29$ (s, $\left.12 \mathrm{H}, \mathrm{SC}\left(\underline{\mathrm{CH}}_{3}\right)_{2} \mathrm{CH}_{2}\right),{ }^{13} \mathrm{C} \mathrm{NMR}\left(75 \mathrm{MHz}, \mathrm{CDCl}_{3}\right)$ $\left.\delta 167.3\left(\mathrm{NH}^{\mathrm{COCH}}\right)_{2}\right), 138.3129 .0,128.8$ and $127.2(\mathrm{ArC}), 48.0\left(\underline{\mathrm{C}} \mathrm{H}_{2} \mathrm{NHCO}\right), 46.6\left(\mathrm{Ph}^{\mathrm{C}} \mathrm{H}_{2} \mathrm{~S}\right), 43.2$ $\left(\mathrm{CO} \underline{C} \mathrm{H}_{2} \mathrm{CO}\right), 33.1\left(\mathrm{~S} \underline{C}\left(\mathrm{CH}_{3}\right)_{2}\right), 26.8\left(\mathrm{SC}\left(\underline{\mathrm{C}} \mathrm{H}_{3}\right)_{2}\right), \mathrm{m} / z(\mathrm{ESI}):[\mathrm{M}+\mathrm{Na}]^{+}$for $\left[\mathrm{C}_{25} \mathrm{H}_{34} \mathrm{~N}_{2} \mathrm{NaO}_{2} \mathrm{~S}_{2}\right]^{+}$ calculated: 481.1959 found: 481.1959 .

Gem-diethyl substituted N,N'-bis(2-benzylthio)ethyl)malonamide derivatives (3a-e)

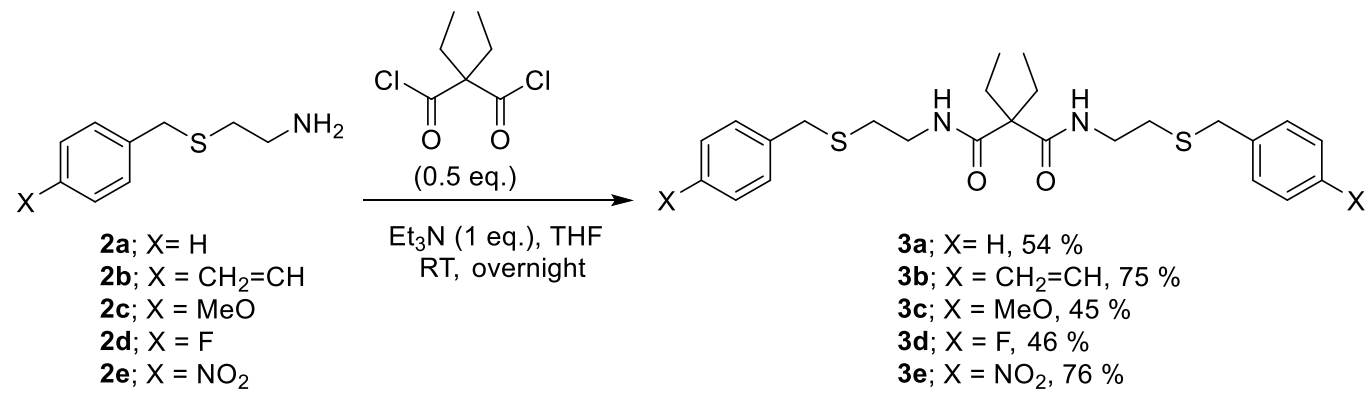

Scheme 4. Syntheses of the gem diethyl substituted malonamide derivatives 3a-e

To a stirred solution of amine (1 eq.) and triethylamine (1 eq.) in dry diethyl ether was added a dry diethyl ether solution of diethyl malonyl chloride $(0.5 \mathrm{eq})$ dropwise for $90 \mathrm{~min}$. The reaction mixture was left to stir overnight (Scheme 4) after which water was added, and the crude product was extracted using diethyl ether. The organic extract was washed successively with $\mathrm{HCl}(2 \mathrm{M}), \mathrm{NaHCO}_{3}$, water and brine after which it was dried over $\mathrm{Na}_{2} \mathrm{SO}_{4}$ and concentrated in vacuo. The crude product was purified by flash chromatography ( $20 \% \mathrm{EtOAc/Hexane}$ to $100 \%$ EtOAc) to give the diethylmalonamide derivatives 3a-e.

N,N'-Bis(2-(benzylthio)ethyl)-2,2-diethylmalonamide (3a). Viscous colourless oil. Yield: $1.1 \mathrm{~g}(54 \%)$, v ( $\mathrm{cm}^{-1}$ ): 3326 (amide N-H stretch), 1630 (amide C=O bend), ${ }^{1} \mathrm{H}$ NMR (300 MHz, $\left.\mathrm{CDCl}_{3}\right) \delta 7.42$ (s, $2 \mathrm{H}, \mathrm{CON} \underline{H C H})_{2}, 7.38-7.12(\mathrm{~m}, 10 \mathrm{H}, \mathrm{ArH}), 3.72$ (s, 4H, SC$\left.\underline{H}_{2} \mathrm{Ph}\right), 3.41$ (m, 4H, NHC $\left.\underline{H}_{2} \mathrm{CH}_{2}\right), 2.57$ (t, 
$\left.J=6.5 \mathrm{~Hz}, 4 \mathrm{H}, \mathrm{CH}_{2} \underline{\mathrm{C}}_{2} \mathrm{~S}\right), 1.85\left(\mathrm{q}, J=7.0 \mathrm{~Hz}, 4 \mathrm{H}, \mathrm{CC}_{2} \mathrm{CH}_{3}\right), 0.82\left(\mathrm{t}, J=7.0 \mathrm{~Hz}, 6 \mathrm{H}, \mathrm{CCH}_{2} \mathrm{C}_{3}\right)$, ${ }^{13} \mathrm{C}$ NMR $\left(75 \mathrm{MHz}, \mathrm{CDCl}_{3}\right) \delta 173.2\left(\underline{\mathrm{COC}}\left(\mathrm{CH}_{2} \mathrm{CH}_{3}\right)_{2} \underline{\mathrm{CO}}\right), 138.2,129.0,128.7$ and $127.3(\mathrm{ArC}), 58.3$ $\left.\left(\mathrm{CO} \underline{C}\left(\mathrm{C}_{2} \mathrm{H}_{5}\right) \mathrm{CO}\right), 38.2\left(\mathrm{NH}^{2} \mathrm{H}_{2} \mathrm{CH}_{2} \mathrm{~S}\right), 35.9\left(\mathrm{CH}_{2} \underline{\mathrm{C}} \mathrm{H}_{2} \mathrm{~S}\right), 31.1\left(\mathrm{~S}_{\underline{C}} \mathrm{H}_{2} \mathrm{Ph}\right), 30.3 \mathrm{C}^{-} \mathrm{H}_{2} \mathrm{CH}_{3}\right), 9.6$ $\left(\mathrm{CCH}_{2} \mathrm{CH}_{3}\right) . \mathrm{m} / z(\mathrm{ESI}):[\mathrm{M}+\mathrm{H}]^{+}$for $\left[\mathrm{C}_{29} \mathrm{H}_{35} \mathrm{~N}_{2} \mathrm{O}_{2} \mathrm{~S}_{2}\right]^{+}$calculated: 459.2140 found: 459.2138 .

N,N'-Bis(2-((4-vinylbenzyl)thio)ethyl)-2,2-diethylmalonamide (3b). Colourless gel. Yield: $0.12 \mathrm{~g}$, (83\%), $v_{\max }\left(\mathrm{cm}^{-1}\right): 3323$ (amide $\mathrm{N}-\mathrm{H}$ stretch), 1655 (amide $\mathrm{C}=\mathrm{O}$ bend), ${ }^{1} \mathrm{H} \mathrm{NMR}\left(300 \mathrm{MHz}, \mathrm{CDCl}_{3}\right) \delta$ $7.36\left(\mathrm{~d}, J=7.5 \mathrm{~Hz}, 4 \mathrm{H}, \mathrm{ArH}\right.$ and $\left.\mathrm{CH}_{2} \mathrm{~N} \underline{H C O}\right), 7.27$ (d, $\left.J=7.5 \mathrm{~Hz}, 4 \mathrm{H}, \mathrm{ArH}\right), 6.69$ (dd, J = 17.5, $11 \mathrm{~Hz}$, $2 \mathrm{H}, \mathrm{CH}_{2} \mathrm{C} \underline{H}_{\mathrm{c}} \mathrm{Ph}$ ), 5.73 (d, J=17.5 Hz, 2H, $\underline{\mathrm{H}}_{\mathrm{a}} \mathrm{H}_{\mathrm{b}} \mathrm{CHPh}$ ), 5.23 (d, J=11.0 Hz, 2H, $\mathrm{CH}_{\mathrm{a}} \underline{H}_{\mathrm{b}} \mathrm{CHPh}$ ), 3.71 (s, 4H, $\mathrm{CH}_{2} \mathrm{CHPhC} \underline{H}_{2} \mathrm{~S}$ ), 3.43 (m, 4H, $\mathrm{CH}_{2} \underline{\mathrm{C}}_{2} \mathrm{NH}$ ), 2.57 (t, $\left.J=6.5 \mathrm{~Hz}, 4 \mathrm{H}, \mathrm{SC}_{2} \mathrm{CH}_{2}\right), 1.85$ (q, $J=$ $\left.7.5 \mathrm{~Hz}, 4 \mathrm{H}, \mathrm{COC}\left(\mathrm{C}_{2} \mathrm{CH}_{3}\right)_{2}\right), 0.82\left(\mathrm{t}, J=7.5 \mathrm{~Hz}, 6 \mathrm{H}, \mathrm{COC}\left(\mathrm{CH}_{2} \underline{\mathrm{C}}_{3}\right)_{2}\right),{ }^{13} \mathrm{C} \mathrm{NMR}\left(75 \mathrm{MHz}, \mathrm{CDCl}_{3}\right) \delta$ $173.2\left(\underline{\mathrm{COC}}\left(\mathrm{CH}_{2} \mathrm{CH}_{3}\right)_{2} \underline{\mathrm{CO}}\right), 137.8$ and $136.7(\mathrm{ArC}), 136.5\left(\mathrm{CH}_{2} \underline{\mathrm{CHPh}}\right), 129.2$ and $126.6(\mathrm{ArC}), 114.0$ $\left.\left(\underline{\mathrm{C}} \mathrm{H}_{2} \mathrm{CHPh}\right), 58.3\left(\mathrm{CO} \underline{C}\left(\mathrm{CH}_{2} \mathrm{CH}_{3}\right)_{2}\right), 38.2\left(\mathrm{CONH}^{2} \mathrm{H}_{2}\right), 35.6\left(\mathrm{CH}_{2} \mathrm{CHPh}^{\mathrm{C}} \mathrm{H}_{2} \mathrm{~S}\right), 31.1\left(\mathrm{C}_{(} \underline{\mathrm{CH}}_{2} \mathrm{CH}_{3}\right)_{2}\right)$, $\left.30.3\left(\mathrm{~S}_{\mathrm{CH}} \mathrm{CH}_{2}\right), 9.6\left(\mathrm{C}_{\left(\mathrm{CH}_{2}\right.} \mathrm{CH}_{3}\right)_{2}\right), m / z(\mathrm{ESI}):[\mathrm{M}+\mathrm{H}]^{+}$for $\left[\mathrm{C}_{29} \mathrm{H}_{36} \mathrm{~N}_{2} \mathrm{O}_{2} \mathrm{~S}_{2}\right]^{+}$calculated: 511.2453 found: 511.2450 .

N,N'-Bis(2-((4-methoxybenzyl)thio)ethyl)-2,2-diethylmalonamide(3c). Viscous colourless oil. Yield: $1.27 \mathrm{~g}(45 \%), v\left(\mathrm{~cm}^{-1}\right): 3337(\mathrm{NH}), 1650$ (amide $\mathrm{C}=\mathrm{O}$ bend), 1240 (C-O; aromatic ether), ${ }^{1} \mathrm{H}$ NMR (300 $\left.\mathrm{MHz}, \mathrm{CDCl}_{3}\right) \delta 7.44\left(\mathrm{~s}, 2 \mathrm{H}, \mathrm{CH}_{2} \mathrm{CH}_{2} \mathrm{~N} \underline{H}\right), 7.23(\mathrm{~d}, J=8.5 \mathrm{~Hz}, 4 \mathrm{H}, \mathrm{ArH}), 6.84(\mathrm{~d}, J=8.5 \mathrm{~Hz}, 4 \mathrm{H}, \mathrm{ArH})$, 3.79 (s, 6H, $\underline{H}_{3} \mathrm{O}$ ), 3.68 (s, 4H, PhC $\underline{H}_{2} \mathrm{~S}$ ), 3.42 (m, 4H, $\mathrm{CH}_{2} \mathrm{C}_{2} \mathrm{NH}$ ), 2.56 (t, $J=6.5 \mathrm{~Hz}, 4 \mathrm{H}$, $\left.\mathrm{SC} \underline{H}_{2} \mathrm{CH}_{2}\right), 1.86\left(\mathrm{q}, J=7.5 \mathrm{~Hz}, 4 \mathrm{H}, \mathrm{CC} \underline{H}_{2} \mathrm{CH}_{3}\right), 0.83\left(\mathrm{t}, J=7.5 \mathrm{~Hz}, 6 \mathrm{H}, \mathrm{CCH}_{2} \underline{C}_{3}\right),{ }^{13} \mathrm{C} \mathrm{NMR}(75 \mathrm{MHz}$, $\left.\mathrm{CDCl}_{3}\right) \delta 173.2$ (NHㅁC), 158.8 (ArC), 130.1 (ArC), 130.0 (ArC), 114.1 (ArC), 58.3 (CO $\underline{C C O}$ ), 55.4 $\left(\mathrm{OCH}_{3}\right), 38.2\left(\mathrm{NH} \underline{C H}_{2} \mathrm{CH}_{2}\right), 35.3\left(\mathrm{~S}_{\underline{C H}} \mathrm{Ph}\right), 31.0\left(\mathrm{C}_{\mathrm{CH}} \mathrm{CH}_{3}\right), 30.2\left(\mathrm{CH}_{2} \underline{C H}_{2} \mathrm{~S}\right), 9.5\left(\mathrm{CCH}_{2} \underline{C} \mathrm{H}_{3}\right), \mathrm{m} / z$ (ESI): $[\mathrm{M}+\mathrm{Na}]^{+}$for $\left[\mathrm{C}_{27} \mathrm{H}_{38} \mathrm{~N}_{2} \mathrm{NaO}_{4} \mathrm{~S}_{2}\right]^{+}$calculated: 518.2171, found: 541.2165.

N,N'-Bis(2-((4-fluorobenzyl)thio)ethyl)-2,2-diethylmalonamide (3d). Viscous yellow oil. Yield: 0.91 $\mathrm{g}(46 \%), \mathrm{v}\left(\mathrm{cm}^{-1}\right): 3296$ (amide $\left.\mathrm{N}-\mathrm{H}\right), 1647$ (amide $\mathrm{C}=\mathrm{O}$ bend), ${ }^{1} \mathrm{H} \mathrm{NMR}\left(300 \mathrm{MHz}, \mathrm{CDCl}_{3}\right) \delta 7.42$ (s, $\left.2 \mathrm{H}, \mathrm{CH}_{2} \mathrm{~N} \underline{H C O}\right), 7.29$ (t, $\left.J=8.0 \mathrm{~Hz}, 4 \mathrm{H}, \mathrm{ArH}\right), 7.00$ (t, $\left.J=8.0 \mathrm{~Hz}, 4 \mathrm{H}, \mathrm{ArH}\right), 3.70$ (s, 4H, SC $\underline{H}_{2} \mathrm{Ph}$ ), $3.44\left(\mathrm{~m}, 4 \mathrm{H}, \mathrm{NHC} \underline{H}_{2} \mathrm{CH}_{2}\right), 2.56\left(\mathrm{t}, J=6.0 \mathrm{~Hz}, 4 \mathrm{H}, \mathrm{CH}_{2} \mathrm{C}_{2} \mathrm{~S}\right.$ ), 1.87 (q, $J=7.0 \mathrm{~Hz}, 4 \mathrm{H}, \mathrm{CC}_{2} \mathrm{CH}_{3}$ ), $0.83\left(\mathrm{t}, J=7.0 \mathrm{~Hz}, 6 \mathrm{H}, \mathrm{CCH}_{2} \underline{\mathrm{C}}_{3}\right) .{ }^{13} \mathrm{C} \mathrm{NMR}\left(75 \mathrm{MHz}, \mathrm{CDCl}_{3}\right) \delta 173.2(\underline{\mathrm{COC}} \underline{\mathrm{CO}}), 162.1\left(\mathrm{~d}, J_{\mathrm{CF}}=242\right.$, $\mathrm{ArC}), 133.9\left(\mathrm{~d}, J_{\mathrm{CCCCF}}=3 \mathrm{~Hz}, \mathrm{ArC}\right), 130.5\left(\mathrm{~d}, J_{\mathrm{CCCF}}=8 \mathrm{~Hz}\right), 115.6\left(\mathrm{~d}, J_{\mathrm{CCF}}=21 \mathrm{~Hz}\right), 58.3(\mathrm{CO} \underline{C C O})$, $38.2\left(\mathrm{NH}_{\mathbf{C}} \mathrm{H}_{2} \mathrm{CH}_{2}\right), 35.1\left(\mathrm{~S} \underline{\mathrm{C}} \mathrm{H}_{2} \mathrm{ArC}\right), 31.1\left(\mathrm{C}_{\mathrm{C}} \mathrm{H}_{2} \mathrm{CH}_{3}\right), 30.3\left(\mathrm{CH}_{2} \underline{\mathrm{CH}} \mathrm{H}_{2} \mathrm{~S}\right), 9.6\left(\mathrm{CCH}_{2} \underline{C} \mathrm{H}_{3}\right) . \mathrm{m} / z(\mathrm{ESI})$ : $[\mathrm{M}+\mathrm{Na}]^{+}$for $\left[\mathrm{C}_{25} \mathrm{H}_{32} \mathrm{~F}_{2} \mathrm{~N}_{2} \mathrm{NaO}_{2} \mathrm{~S}_{2}\right]^{+}$calculated; 517.1771 found; 517.1765.

N,N'-2,2-Diethyl-bis(2-((4-nitrobenzyl)thio)ethyl)malonamide (3e). Viscous yellow oil. Yield: $2.84 \mathrm{~g}$ (76\%), v(cm $\left.{ }^{-1}\right): 3294$ (N-H stretch), 1647 (amide C=O bend), 1544 and 1367 (N-O stretch), ${ }^{1} \mathrm{H}$ NMR $\left(300 \mathrm{MHz}, \mathrm{CDCl}_{3}\right) \delta 8.18(\mathrm{~d}, J=8.5 \mathrm{~Hz}, 4 \mathrm{H}, \mathrm{ArH}), 7.51$ (d, $\left.J=8.5 \mathrm{~Hz}, 4 \mathrm{H}, \mathrm{ArH}\right), 7.45$ (s, 2H, $\mathrm{CH}_{2} \mathrm{CH}_{2} \mathrm{~N} \underline{H}$ ), 3.81 (s, $4 \mathrm{H}, \mathrm{PhC}_{2} \mathrm{~S}$ ), $3.46\left(\mathrm{~m}, 4 \mathrm{H}, \mathrm{SCH}_{2} \mathrm{C}_{2}\right.$ ), 2.57 (t, J=6.5 Hz, 4H, SC $\underline{H}_{2} \mathrm{CH}_{2}$ ), 1.87 (q, $\left.J=7.5 \mathrm{~Hz}, 4 \mathrm{H}, \mathrm{CC}_{\mathrm{H}_{2}} \mathrm{CH}_{3}\right), 0.83\left(\mathrm{t}, J=7.5 \mathrm{~Hz}, 6 \mathrm{H}, \mathrm{CCH}_{2} \mathrm{C}_{3}\right),{ }^{13} \mathrm{C} \mathrm{NMR}\left(75 \mathrm{MHz}, \mathrm{CDCl}_{3}\right) \delta 173.3$ 


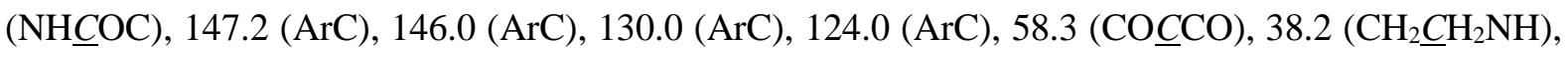
$35.3\left(\mathrm{Ph} \underline{\mathrm{C}} \mathrm{H}_{2} \mathrm{~S}\right), 31.3\left(\mathrm{C}_{\underline{C}} \mathrm{H}_{2} \mathrm{CH}_{3}\right), 30.5\left(\mathrm{~S}_{\underline{C}} \mathrm{CH}_{2} \mathrm{CH}_{2}\right), 9.6\left(\mathrm{CCH}_{2} \underline{C} \mathrm{H}_{3}\right), \mathrm{m} / z(\mathrm{ESI}):[\mathrm{M}+\mathrm{Na}]^{+}$for $\left[\mathrm{C}_{25} \mathrm{H}_{32} \mathrm{~N}_{4} \mathrm{NaO}_{6} \mathrm{~S}_{2}\right]^{+}$calculated: 571.1661, found 571.1605.

Silver complexes $[\mathrm{Ag}(\mathrm{Ia})] \mathrm{OSO}_{2} \mathrm{CF}_{3}(4 a-c)$

$$
\text { 1a } \underset{\text { EtOAc, reflux, } 15 \text { min }}{\stackrel{\mathrm{AgOSO}_{2} \mathrm{CF}_{3}(1-3 \text { eq. })}{\longrightarrow}}\left[\mathrm{Ag}_{x}(\mathbf{1 a})\right]^{\mathrm{n+}}
$$

Scheme 5. Synthesis of $\mathrm{Ag}^{+}-1 \mathrm{a}$ complexes 4a-c

The $\mathrm{Ag}^{+}-\mathbf{1 a}$ complexes $\mathbf{4 a - c}$ were accessed following the method reported by Daubinet.[12] Briefly, 1a (1 eq.) was dissolved in EtOAc (5 ml) and added to $\mathrm{AgOSO}_{2} \mathrm{CF}_{3}$ (1, 2 or 3 eq.) also in EtOAc (5 ml) and heated at reflux for $15 \mathrm{~min}$ (Scheme 5). The product was concentrated in vacuo and, samples suitable for mass spectrometry analysis were prepared.

$[\mathrm{Ag}(\mathrm{Ia})] \mathrm{OSO}_{2} \mathrm{CF}_{3}(4 a-c)$

4a: Brown paste. Yield: $30 \mathrm{mg}$ (91 \%), 4b: Brown solid. Yield: $30 \mathrm{mg}(65 \%)$, 4c: Yellow paste. Yield: $50 \mathrm{mg}(85 \%) . \mathrm{m} / \mathrm{z}$ (ESI): $[\mathrm{M}+\mathrm{Ag}]^{+}$calculated for $\left[\mathrm{C}_{21} \mathrm{H}_{26} \mathrm{AgN}_{2} \mathrm{O}_{2} \mathrm{~S}_{2}\right]^{+} ; 509.0487$ found; 509.0481 .

\section{Selective $\mathrm{Ag}^{+}$extraction studies[13]}

A solution of equal concentration of $\mathrm{Cu}^{2+}, \mathrm{Ag}^{+}$and $\mathrm{Pb}^{2+}$ (4 ppm each) in $0.023 \mathrm{M} \mathrm{Na}_{2} \mathrm{SO}_{4}$ in $0.476 \mathrm{M}$ $\mathrm{HNO}_{3}$ was prepared from a $500 \mathrm{ppm}$ stock solution. Chloroform was presaturated with twice its volume of $0.5 \mathrm{M} \mathrm{H}_{2} \mathrm{SO}_{4}$ by constant shaking in a separatory funnel for $5 \mathrm{~min}$. The chloroform was used to prepare the malonamide derivative ligand solutions with molarity equal to 250 times that of $\mathrm{Ag}^{+}$were prepared. For each metal extraction experiment, equal volumes $(10 \mathrm{ml})$ of the metal solution and the ligand solution were contacted by rapid and vigorous stirring (using a stir bar) for $15 \mathrm{~min}$ in a capped plastic vial partially immersed in an oil bath set at $25^{\circ} \mathrm{C}$. Each experiment was undertaken in duplicate. After stirring, the immiscible solutions were transferred to a separatory funnel, allowed to separate and collected separately. The aqueous layer was collected into a beaker and the residual chloroform was removed over a steam bath in about $15 \mathrm{~min}$. Then the aqueous layer was made back up to $10 \mathrm{ml}$ and prepared for ICP-OES analysis. The metal extraction efficiencies were determined using;

$\% E E=\frac{C_{i}-C_{f}}{C_{i}} X 100$

Where $\% E E$ is the percentage extraction efficiency, $C_{i}$ and $C_{f}$ are the initial and final metal ion concentrations respectively.

The selectivity of the ligands for $\mathrm{Ag}^{+}$relative to $\mathrm{Cu}^{2+}$ and $\mathrm{Pb}^{2+}$ was determined as follows[14]; 


$$
K_{A g^{+}} /_{M^{n+}}=\frac{D_{A g^{+}}}{D_{M^{n+}}}
$$

Where $K_{\mathrm{Ag}^{+}} /_{\mathrm{M}^{n+}}$ represents the selectivity coefficient of $\mathrm{Ag}^{+}$relative to $\mathrm{M}^{\mathrm{n+}}\left(\mathrm{M}^{\mathrm{n}+}=\mathrm{Cu}^{2+}\right.$ or $\left.\mathrm{Pb}^{2+}\right), D$ is the distribution coefficient of the metal ions between the aqueous and the organic phases defined as;

$D_{M^{n+}}=\frac{C_{i}-C_{f}}{C_{f}} \times \frac{V_{a q}}{V_{\text {org }}}$

$D_{M}{ }^{n+}$ represents the distribution coefficient for the metal ion $\mathrm{M}^{\mathrm{n}+}, C_{i}$ and $C_{f}$ are initial and the final metal ion concentrations, $V_{a q}$ and $V_{\text {org }}$ are the volumes of the aqueous and the organic solutions respectively.

Since equal volumes of the aqueous and the organic solutions were used, the distribution factor formula was simplified into;

$D_{M^{n+}}=\frac{C_{i}-C_{f}}{C_{f}}$

\section{Job's plot for the ${ }^{1} \mathrm{H}$ NMR titration of $\mathrm{AgClO}_{4}$ against 1a. [15]}

Standard solutions of equal concentrations $(0.005 \mathrm{M})$ of ligand $\mathbf{1 a}$ and $\mathrm{AgClO}_{4}$ were prepared separately in $\mathrm{d}_{6}$-DMSO. Varying ratios of each solution $\left(\mathbf{1 a}\right.$ and $\left.\mathrm{AgClO}_{4}\right)$ were then taken and mixed together (with the overall concentration remaining the same) to target different $\mathrm{Ag}^{+}$mole fractions $\left(\chi_{\mathrm{Ag}^{+}}{ }^{+}\right.$). For example, to target a $\chi_{\mathrm{Ag}^{+}}{ }^{+}=0.1$, a $0.45 \mathrm{ml} 1 \mathbf{a}$ solution was mixed with a $0.05 \mathrm{ml} \mathrm{AgClO}_{4}$ solution inside an amber-colored NMR tube. Also, to target a $\chi_{\mathrm{Ag}^{+}}{ }^{+}=0.2$, a $0.40 \mathrm{ml}$ 1a solution was mixed with a 0.10 $\mathrm{ml} \mathrm{AgClO}_{4}$ solution and so on until a $\chi_{\mathrm{Ag}^{+}}{ }^{+}=0.9$ was prepared by mixing a $0.05 \mathrm{ml}$ 1a solution with a $0.45 \mathrm{ml} \mathrm{AgClO}_{4}$ solution. The ${ }^{1} \mathrm{H}$ NMR experiments of all mixtures and the free ligand 1a were recorded. The plot of $\chi_{\mathbf{1 a}} \Delta \delta$ (where $\chi_{\mathbf{1 a}}=$ mole fraction of $\mathbf{1 a}$ and $\Delta \delta=$ chemical shift of $\mathrm{Ag}^{+}-\mathbf{1} \mathbf{a}$ complex minus chemical shift of free 1a) against $\chi_{\mathrm{Ag}^{+}}{ }^{+}$was constructed from the data generated.

\section{Mole ratio plot for the ${ }^{1} \mathrm{H}$ NMR titration of $\mathrm{AgClO}_{4}$ against 1a[15]}

The ligand 1a (13 mg, $0.03 \mathrm{mmol}$ ) was dissolved in $0.5 \mathrm{ml} \mathrm{d} 6$-DMSO and transferred into an ambercolored NMR tube. Standard solution of $\mathrm{AgClO}_{4}(0.539 \mathrm{M})$ was made up in $\mathrm{d}_{6}$-DMSO and constant volume $(30 \mu \mathrm{L})$ of the $\mathrm{AgClO}_{4}$ solution was added to $\mathbf{1 a}$ in the NMR tube to target varying $\mathrm{Ag}^{+} / \mathbf{1 a}$ mole ratios $\left(n_{\mathrm{Ag}}{ }^{+} / n_{1 \mathrm{a}}=0.5\right.$ to 4.0$)$. For example, to target a $n_{\mathrm{Ag}}{ }^{+} / n_{1 \mathrm{a}}=0.5$, a $30 \mu \mathrm{L}$ of the $\mathrm{AgClO}_{4}$ solution was added using a glass syringe to the neat 1a solution and the ${ }^{1} \mathrm{H}$ NMR spectrum was recorded. Also, to target a $n_{\mathrm{Ag}}{ }^{+} / n_{1 \mathbf{a}}=1.0$, a $30 \mu \mathrm{L} \mathrm{AgClO}_{4}$ solution was added to the $\mathrm{AgClO}_{4}-\mathbf{1 a}$ with $n_{\mathrm{Ag}}{ }^{+} / n_{1 \mathbf{a}}=0.5$ and the ${ }^{1} \mathrm{H}$ NMR spectrum was recorded. The constant addition of the $\mathrm{AgClO}_{4}$ solution was continued until 
a $n_{\mathrm{Ag}}{ }^{+} / n_{1 \mathrm{a}}=4.0$ was achieved. The plot of $\Delta \delta$ (where $\Delta \delta=$ chemical shift of $\mathrm{Ag}^{+}-\mathbf{1 a}$ complex minus chemical shift of free 1a) against $n_{\mathrm{Ag}}{ }^{+} / n_{1 \mathrm{a}}$ was constructed using the data generated.

\section{Results and Discussion}

\section{Syntheses of $S$-benzylcysteamines (2a-e) and gem-disubstituted $S$-benzylcysteamine $2 \mathrm{~g}$}

The $S$-benzylcysteamines 2a-e, were accessed following a modified protocol to that reported by Ghosh and Tochtrop.[10] For the amine 2a, it was necessary to shorten the reaction time from 40 minutes to 20 minutes in order to minimize the formation of the dibenzylated product $\mathbf{2 h}$.

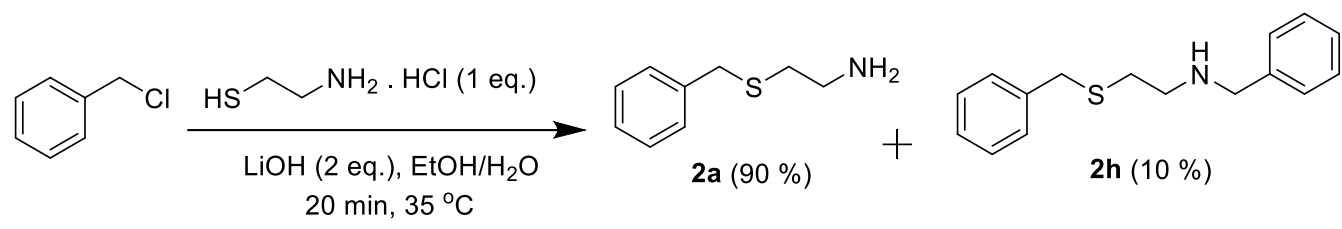

Scheme 6. Syntheses of $S$-benzylcysteamine $\mathbf{2 a}$ showing the dibenzylated product $\mathbf{2 h}$

However, for the other amines $\mathbf{2 b}$-e it was necessary to extend the reaction time to $40 \mathrm{~min}$ to give good selectivity ( $<3 \%$ dibenzylated products observed as evidenced from ${ }^{1} \mathrm{H}$ NMR). Using these reaction durations (20 $\mathrm{min}$ for $\mathbf{2 a}$ and $40 \mathrm{~min}$ for $\mathbf{2 b} \mathbf{b}$-e) the desired compounds apart from $\mathbf{2 d}$ were prepared without the need for further purification (as evidenced by their ${ }^{1} \mathrm{H}$ NMR spectra). These amines served as starting material for the synthesis of the malonamide derivatives $\mathbf{1 a}$ and $\mathbf{1 e - h}$.

In order to assess the effect of sterics on potential novel malonamide derived ligands, the substituted $S$ benzylcysteamine $\mathbf{2} \mathbf{g}$ was synthesized by a modification of a method reported by Caroll et al.[11]. The poor yield ( $23 \%$ ) obtained for $\mathbf{2 g}$ was attributed to the loss of acetone, during the reaction

In the original published procedure by Daubinet and Kaye[9], access to the ligand 1a required a reaction duration of 7 days, at the end of which a yield of $24 \%$ was obtained. We prepared the ligand 1a in less than 1 day with an improved yield of $33 \%$ through an alternative pathway involving the dropwise addition of 1 equivalent of malonyl chloride to 4 equivalents of the amine $\mathbf{2 a}$ in dry THF. Related ligands 1e-i were accessed (in low to high yields) using the same protocol.

The gem-diethyl malonamide derivatives 3a-e were obtained in much better yields $(46-75 \%)$ than those obtained for the unsubstituted malonamide derivatives (1a and 1e-i), presumably due to removal of competing enolisation pathways that would lower the yields.

\section{$\mathrm{Ag}^{+}$extraction studies - electronic and steric effects}

Extraction studies in chloroform following the method reported by Sole and Hiskey[13] were carried out to assess the selectivity and efficiency of $\mathrm{Ag}^{+}$extraction by the malonamide derived ligands $\mathbf{1 a}$, $1 \mathbf{e}-$ 
$\mathbf{h}$ and 3a prepared. Prior to its use in preparation of the ligand solutions, the chloroform solvent was presaturated with twice its volume of an acidified deionized water to remove any water-soluble components in order to minimize volume changes during the liquid/liquid metal extraction process. An aqueous solution containing 4 ppm each of $\mathrm{Ag}^{+}, \mathrm{Cu}^{2+}$ and $\mathrm{Pb}^{2+}$ in $0.0232 \mathrm{M} \mathrm{NaNO}_{3}$ was prepared by dilution of a stock solution. The choice of $\mathrm{Cu}^{2+}$ and $\mathrm{Pb}^{2+}$ as competing ions for the efficiency and selectivity extractions is borne out of the knowledge that these metals usually coexist with $\mathrm{Ag}^{+}$in ores and mine tailings, for instance.[16]. We decided to prepare mixed metal aqueous solutions with very low concentrations (4 ppm) of $\mathrm{Ag}^{+}, \mathrm{Cu}^{2+}$ and $\mathrm{Pb}^{2+}$ because $\mathrm{Ag}^{+}$typically exists in very low concentrations in $\mathrm{Ag}^{+}$repositories where our novel malonamide derived ligands may be applied.[16] Furthermore, $\mathrm{NaNO}_{3}$ was added to maintain the ionic strength of the aqueous phase during extraction. For sensible comparisons to be made, each solvent extraction experiment was undertaken in duplicate and with vigorous stirring (using a stir bar with equal volumes of ligand and metal solutions for 15 mins). The concentration of the metals in the raffinate was measured by means of ICP-OES and each metal concentration value from the ICP-OES was the average of three analytic runs.

\section{Control study}

A control experiment was undertaken with the metal solution using the chloroform solvent without any ligand present. This would determine any extraction efficiency of the solvent itself (the background extraction). Interestingly, we observed that the neat chloroform itself (the control) was able to extract some metals with a slight preference for $\mathrm{Ag}^{+}$(Figure 3). This is likely due to the differential partial solubility of these metals in chloroform. Notwithstanding, the extraction efficiency of the ligand 1a for $\mathrm{Ag}^{+}$was significantly higher than that of the neat chloroform (Figure 3), underlining the importance of the ligand 1a in efficient $\mathrm{Ag}^{+}$extraction.

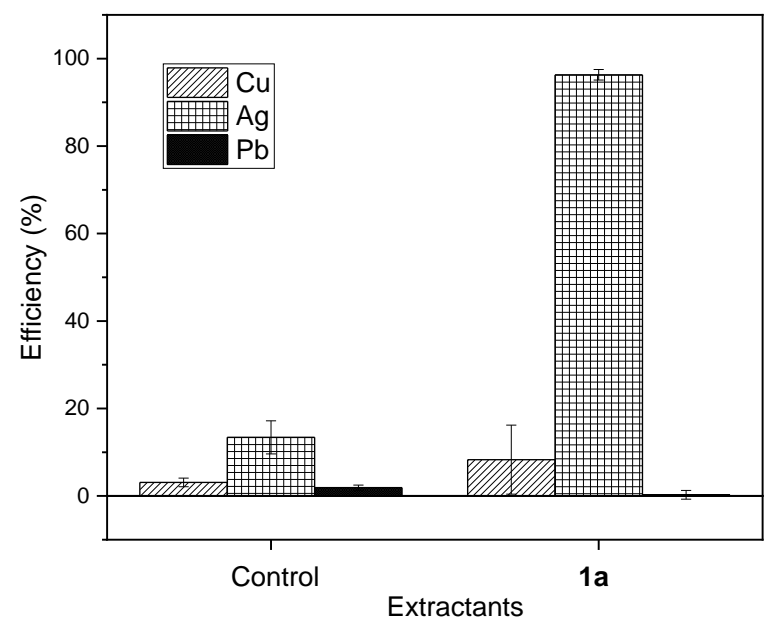


Figure 3. ICP-OES data showing metal extraction efficiencies by the neat chloroform (control) and the malonamide derived ligand 1a

\section{Electronics effect}

The effect of electronic differences in the aryl grouping of the malonamide derived ligands on the efficiency of $\mathrm{Ag}^{+}$recovery was studied by comparing extraction efficiencies and selectivity's exhibited by the ligands 1a and 1e-h. The observed extraction efficiencies are presented in Figure 4.

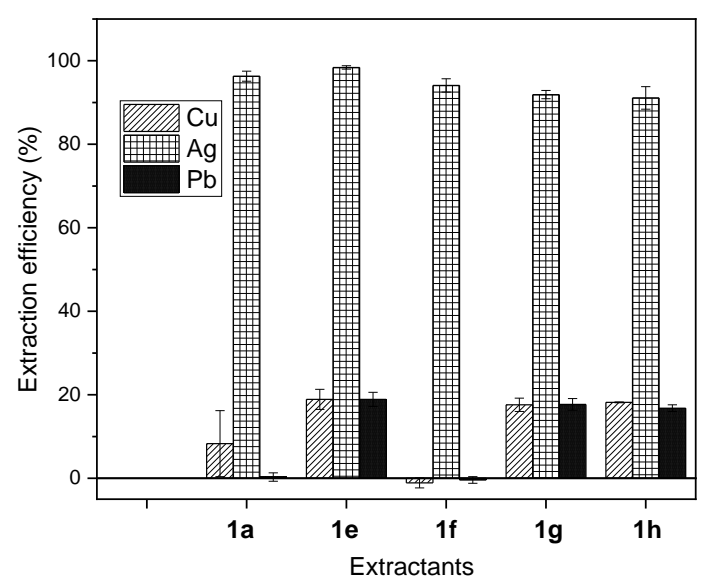

Figure 4. ICP-OES data showing metal extraction efficiencies by the malonamide derived ligands 1a, 1e-h

While the efficiencies for $\mathrm{Ag}^{+}$extraction by these ligands differ only slightly; $\mathbf{1 a}=96.3 \pm 1.2 \%, \mathbf{1 e}=$ $98.4 \pm 0.4 \%, \mathbf{1 f}=94.1 \pm 1.6 \%, \mathbf{1 g}=91.1 \pm 1.0 \%, \mathbf{1 h}=91.1 \pm 2.7 \%$ (Figure 4 ) extraction efficiencies for those with electron withdrawing substituents $\mathbf{1 g}$ and $\mathbf{1 h}$ are the lowest (extraction efficiency order $\mathbf{1 h}=$ 1g $<$ 1f $<$ 1a $<$ 1e) $($ Table 1$)$. The highest extraction efficiency observed for 1e could be because it would give the least polar $\mathrm{Ag}^{+}$complex and thus have the highest solubility in chloroform.[17] Correspondingly, the least extraction efficiencies were observed for $\mathbf{1 g}(\mathrm{X}=\mathrm{F})$ and $\mathbf{1 h}\left(\mathrm{X}=\mathrm{NO}_{2}\right)$ because (a) the $\mathrm{Ag}^{+}$complexes are the most polar and so less soluble in chloroform[17] and (b) the electron withdrawing nature of the aryl groups may have made the sulfur atom lone pairs less available for binding for $\mathrm{Ag}^{+}$(the soft sulfur atom being crucial in efficiency and selectivity following the HSAB rule).[18] For the methoxy analogue $\mathbf{1 f}$ a combination of the polar nature of the $\mathrm{Ag}^{+}$complex lowering extraction efficiency with the more electron rich nature of the sulfur atom increasing binding leads to an intermediate extraction efficacy. The latter explanation (b), if true, would also explain the poorer selectivity's of the ligands with electron withdrawing groups $(\mathbf{1 e}, \mathbf{1 g}, \mathbf{1 h})$, noting that in the styryl analogue 1e the inductive electron withdrawing nature of the vinyl group will be the dominant effect. 
A greater selectivity would be predicated and is in fact observed for the strongly electron donating methoxy substituent in $\mathbf{1 f}$, where selectivity verses $\mathrm{Cu}^{2+}$ is approximately nine times better than $\mathbf{1 a}$. The results observed here suggest that electronics have only a small influence on the efficiency of $\mathrm{Ag}^{+}$ extraction by these ligands but a significant effect on selectivity.

Table 1. Efficiency and selectivity of malonamide derived ligands $\mathbf{1 a}, \mathbf{1 e - h}$ for $\mathrm{Ag}^{+}$relative to

$$
\mathrm{Cu}^{2+} \text { and } \mathrm{Pb}^{2+}
$$

\begin{tabular}{cccc}
\hline Ligand & $\begin{array}{c}\text { Efficiency } \\
(\boldsymbol{\%})\end{array}$ & $\mathbf{K}_{\mathbf{A g}^{+}} / \mathbf{C u}^{2+}$ & $\mathbf{K}_{\mathbf{A g}^{+}} / \mathbf{P b}^{2+}$ \\
\hline $\mathbf{1 a}$ & $96.3 \pm 1.2$ & 11.6 & 321 \\
$\mathbf{1 e}$ & $98.4 \pm 0.4$ & 5.2 & 5.2 \\
$\mathbf{1 f}$ & $94.1 \pm 1.6$ & 85.5 & 235.3 \\
$\mathbf{1 g}$ & $91.1 \pm 1.0$ & 5.2 & 5.2 \\
$\mathbf{1 h}$ & $91.1 \pm 2.7$ & 5.0 & 5.4 \\
\hline
\end{tabular}

\section{Steric effect}

The effect of steric hindrance at various sites in the malonamide derived ligands was investigated. Steric hindrance around the crucial sulphur atom required for binding in ligand $\mathbf{1 i}$ might be predicted to lead to lower efficiencies in extraction while placing steric hindrance further away at the central carbon represented by ligand $\mathbf{3 a}$, might be predicted to have less effect (Figure 5).

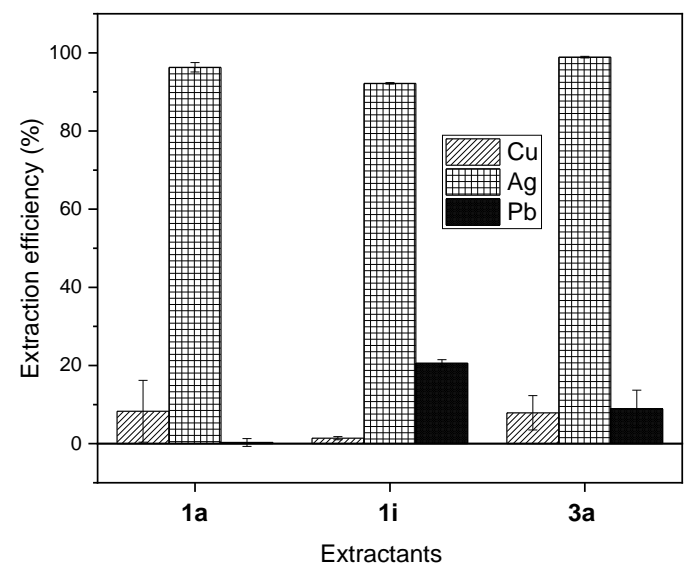

Figure 5. ICP-OES data showing metal extraction efficiencies by malonamide derived ligands 1a, 1i and 3a

Ligand 1i, sterically hindered near the sulfur atom, extracted with less efficiency ( 4\%) for $\mathrm{Ag}^{+}$ $(92.2 \pm 0.2 \%)$ than the analogue without such steric hindrance 1a $(96.3 \pm 1.2 \%)$, (Figure 5) as predicted. 
This is attributed to the slightly greater difficulty of $\mathbf{1 i}$ in assuming the right conformation needed to bind $\mathrm{Ag}^{+}$because of the presence of the dimethyl substitution.[19] On the other hand, malonamide 3a, sterically hindered at the acyl region extracted slightly higher amounts of $\mathrm{Ag}^{+}$compared to $1 \mathrm{a}$ attributed to the higher solubility of the $\mathrm{Ag}^{+}-\mathbf{3 a}$ complex in chloroform than the $\mathrm{Ag}^{+}-\mathbf{1 a}$ complex $(\mathbf{1 a}=96.3 \pm 1.2$ $\%$ verses $\mathbf{3 a}=98.9 \pm 0.2 \%$ ). It is worth noting that the ligand $\mathbf{3 a}$ extracted slightly higher amounts of $\mathrm{Ag}^{+}$than the ligand 1i, attributed to the location of steric hindrance away from the crucial sulfur donor centre as earlier explained. Interestingly both $\mathbf{1} \mathbf{i}$ and $\mathbf{3 a}$ were less selective than $\mathbf{1 a}$ for binding with $\mathrm{Pb}^{2+}$

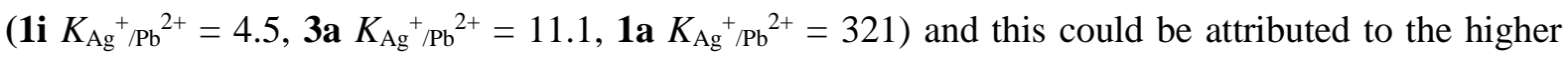
solubility of all the malonamide- $\mathrm{Pb}^{2+}$ complexes in chloroform due to the gem dialkyl groupings.[17]

\section{Investigation of $\mathrm{Ag}^{+}$-malonamide derivative 1a binding stoichiometry}

\section{Mass spectrometry studies}

To gain insight into the nature of the binding of $\mathrm{Ag}^{+}$by the malonamide derived ligands, $\mathrm{Ag}^{+}$complexes of 1a, were prepared by varying the equivalents of the silver salt (1-3 eq) relative to the ligand. Consequently, the $\mathrm{Ag}^{+}-\mathbf{1 a}$ complexes (4a-c) were obtained in moderate to high yields (Table 2) and characterized by means of low- and high-resolution mass spectrometry. In the wide scan $(\mathrm{m} / z 0-2400)$ low resolution mass spectrometry (LRMS) of $\mathbf{4 a}$, the only pair of peaks with ratio of $\sim 1: 1$ (indicative of a $\mathrm{Ag}^{+}$complex) were observed at $\mathrm{m} / z 509$ and 511 (Figure 6). Indeed, the $\mathrm{m} / \mathrm{z} 509$ was confirmed by high resolution mass spectrometry (HRMS) analyses as the complex - $\left[{ }^{107} \mathrm{Ag}(\mathbf{1 a})\right]^{+}$(found: 509.0481, calculated: 509.0487). Interestingly, reacting the ligand 1a with 2 equivalents and 3 equivalents of silver triflate gave $\mathbf{4 b}$ and $\mathbf{4 c}$ respectively (Entries 2 and 3, Table 2) which were also both found, after LRMS analyses to be $\mathrm{Ag}^{+}-\mathbf{1 a}$ complexes with a 1:1 stoichiometry. These results indicate that the stoichiometry of the $\mathrm{Ag}^{+}$complex with malonamide $\mathbf{1 a}$ is perhaps 1:1.

Table 2. Effect of $\mathrm{Ag}^{+}$equivalent on stoichiometry of $\mathrm{Ag}^{+}$complexes of $\mathbf{1 a}$ and silver triflate

\begin{tabular}{cccccc}
\hline Entry & Ligand & $\begin{array}{c}\mathbf{A g O S O}_{2} \mathbf{C F}_{3} \\
\text { eq. }\end{array}$ & Product & $\mathbf{A g}^{+}: \mathbf{1 a}$ & Yield \\
\hline 1 & $\mathbf{1 a}$ & 1 & $\mathbf{4 a}$ & $1: 1$ & $91 \%$ \\
2 & $\mathbf{1 a}$ & 2 & $\mathbf{4 b}$ & $1: 1$ & $65 \%$ \\
3 & $\mathbf{1 a}$ & 3 & $\mathbf{4 c}$ & $1: 1$ & $85 \%$ \\
\hline
\end{tabular}




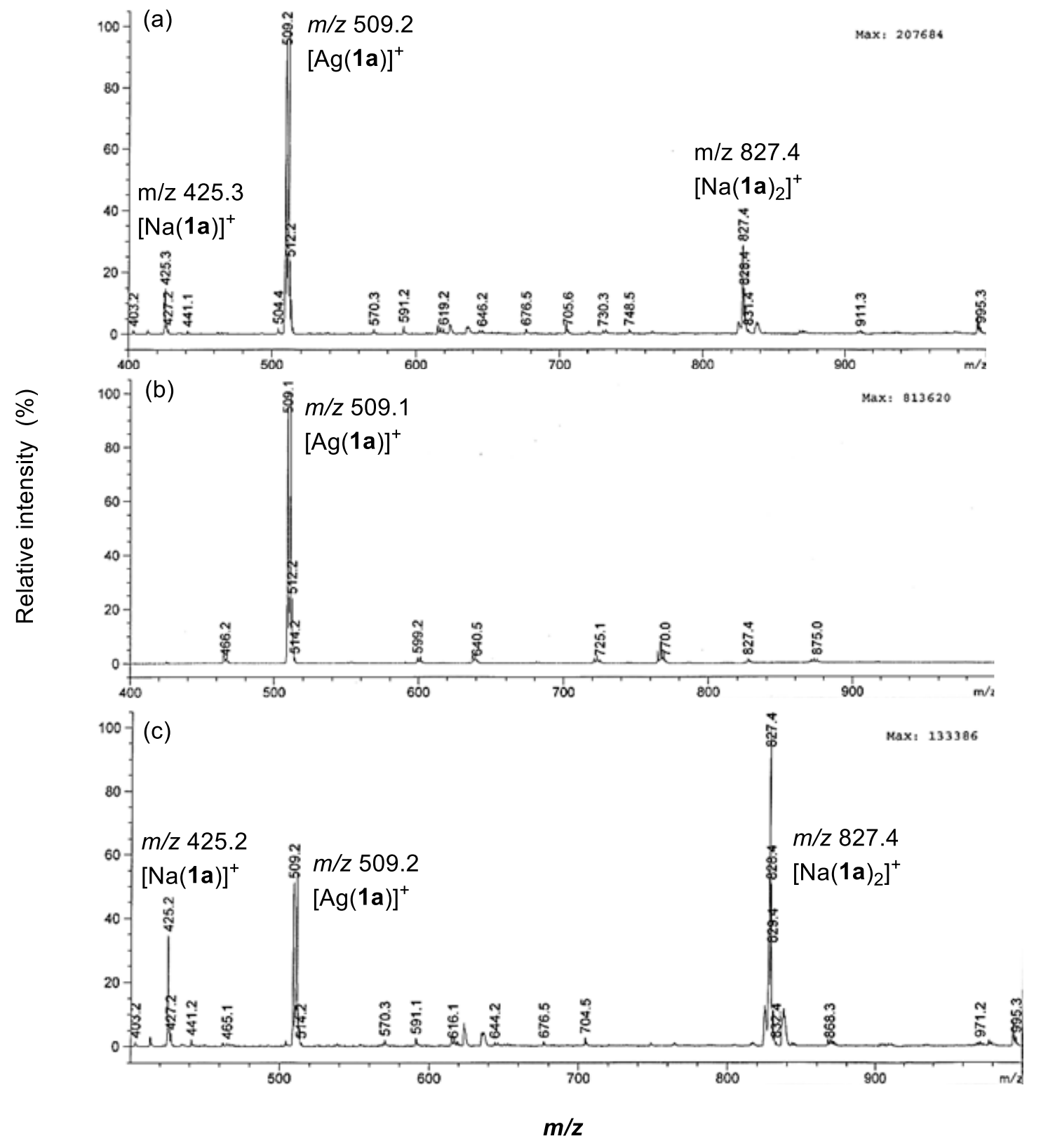

Figure 6. Partial low-resolution ESI mass spectra of $\mathrm{Ag}^{+}-\mathbf{1 a}$ complexes from treatment of $\mathbf{1 a}$ with (a) one (b) two and (c) three equivalents of $\mathrm{AgOSO}_{2} \mathrm{CF}_{3}$

\section{Proton NMR studies - Job's plot and mole ratio}

The Job's plot for the $\mathrm{Ag}^{+}-\mathbf{1 a}$ perchlorate complex was constructed from ${ }^{1} \mathrm{H}$ NMR titration of equal concentrations $(0.005 \mathrm{M})$ of ligand $1 \mathrm{a}$ against $\mathrm{AgClO}_{4}$. The ${ }^{1} \mathrm{H}$ NMR titration of the $\mathrm{Ag}^{+}$against the ligand 1a was undertaken in DMSO- $\mathrm{d}_{6}$ after initial solvent screening experiments revealed the unsuitability of other common NMR solvents tested (due to poor solubility of the ligand 1a). The ${ }^{1} \mathrm{H}$ NMR titrations of $\mathrm{Ag}^{+}$against $\mathbf{1 a}$ did not lead to the appearance of new peaks (Figure 7a) but caused 
distinct changes in chemical shifts, indicating a fast rate of $\mathrm{Ag}^{+}$exchange between the complexed and uncomplexed states. The Job's plot for the interaction of $\mathrm{Ag}^{+}$with $\mathbf{1 a}$ (Figure $7 \mathrm{~b}$ ) was constructed after appropriate calculations had been undertaken. The gentle curvature of the curves in the Job's plot (Figure 7b) indicates the binding between the ligand $\mathbf{1 a}$ and $\mathrm{Ag}^{+}$is perhaps weak.[20] Furthermore, it can be observed that the coefficient on the $\mathrm{x}$-axis of the maxima of all curves in the Job's plot is at $\chi_{\mathrm{Ag}+}$ $=0.5$, indicating that the malonamide $\mathbf{1 a}$ bind $\mathrm{Ag}^{+}$in any of the 1:1,2:2 or any other $n: n$ fashion. To confirm the actual stoichiometry, a different ${ }^{1} \mathrm{H}$ NMR titration of the interaction of $1 \mathrm{a}$ with $\mathrm{Ag}^{+}$were constructed towards generating a mole ratio plot. Therefore, a constant amount of a $0.539 \mathrm{M} \mathrm{Ag}^{+}$ solution was successively added to the ligand $\mathbf{1 a}$ solution to prepare $\mathrm{Ag}^{+} / \mathbf{1} \mathbf{a}$ mole ratios ranging from 0.5 to 4.0. Proton NMR spectra after successive addition of the $\mathrm{Ag}^{+}$solution were recorded (Figure 7c), and the mole ratio plot was constructed from the values obtained (Figure 7d). In a typical mole ratio plot, the stoichiometry of a complex is the coefficient on the x-axis of the point of inflection. The point of inflection is the point on a curve where the shape of the curve changes. In the case of the $\mathrm{Ag}^{+}-\mathbf{1 a}$ complex (Figure 7d), it was very difficult to get a distinct point of inflection for any of the curves, indicating a weak binding between the $\mathrm{Ag}^{+}$and $\mathbf{1 a}$ and confirming the observation from the Job's plot.[20] It also suggests that the $\mathrm{Ag}^{+}-\mathbf{1 a}$ complex may be adopting more than one type of stoichiometry in solution including the 1:1 observed from mass spectrometry and the Job's plot. 


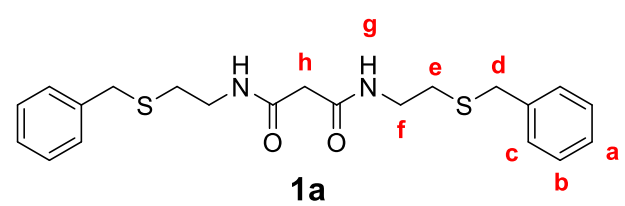

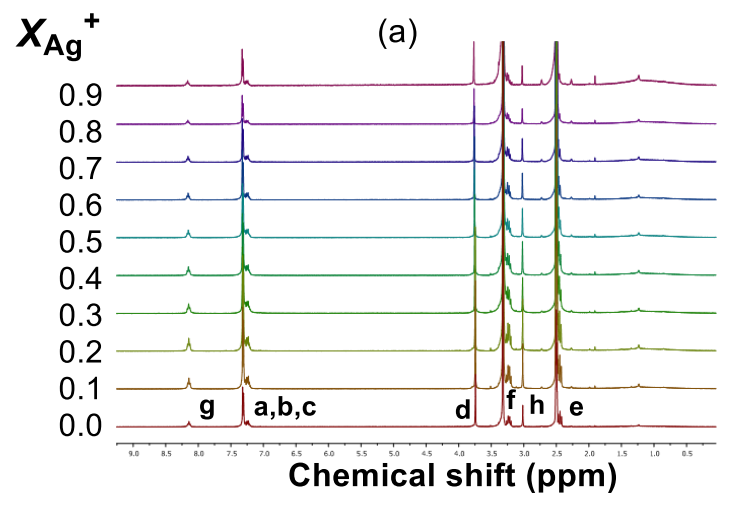

(c)

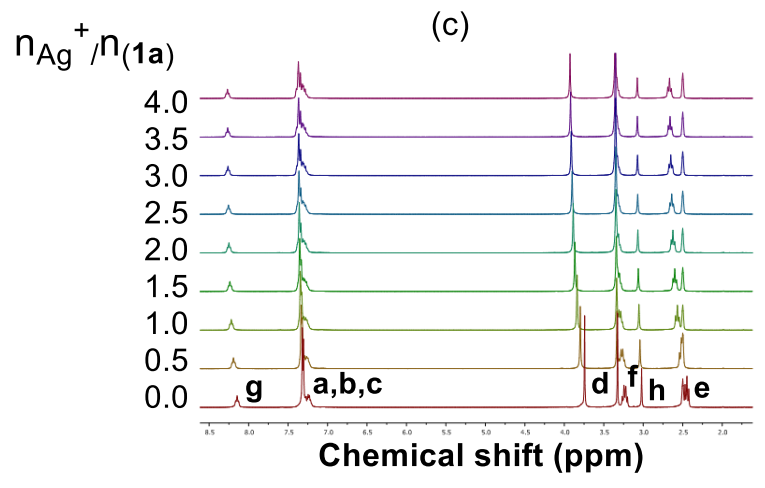

(b)

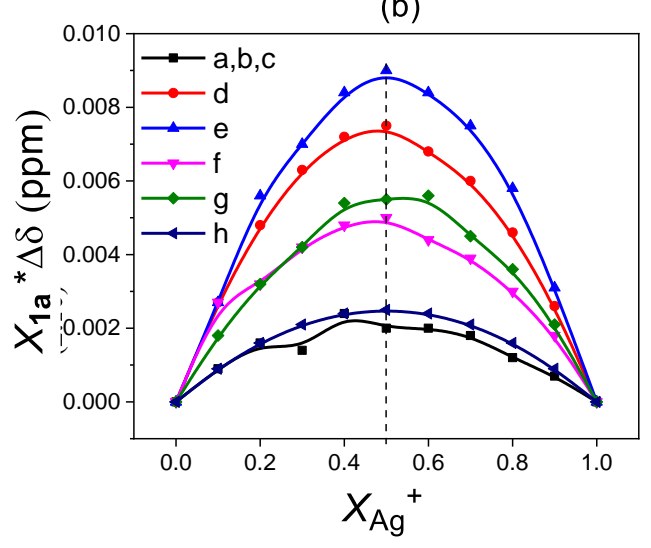

(d)

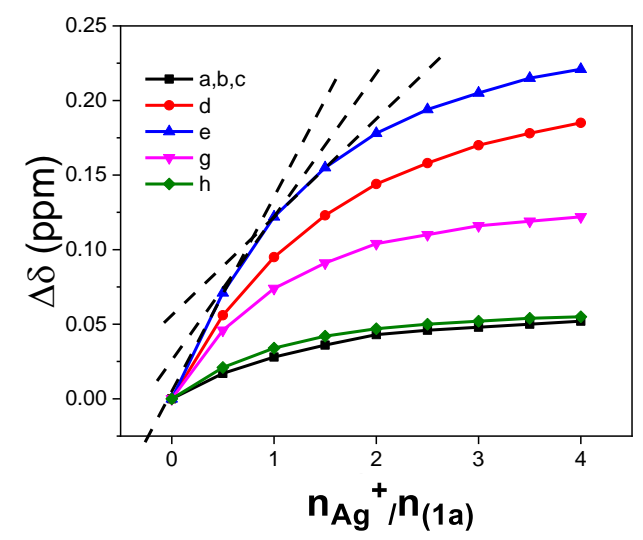

Figure 7. (a and c) ${ }^{1} \mathrm{H}$ NMR (300 MHz) spectra of the interaction of $\mathrm{Ag}^{+}$with 1a, (b) Job's and (d) mole ratio plots of the interaction of $\mathrm{Ag}^{+}$with ligand $\mathbf{1 a}$.

In the Job's plot (Figure $7 \mathrm{~b}$ ), the highest $\Delta \delta$ values were observed for protons 'e' and 'd' in 1a (the protons on carbons alpha to the $S$ - donor atoms). The next highest $\Delta \delta$ values were observed for protons ' $\mathrm{g}$ ' in $\mathbf{1 a}$ (the amide protons). The observed high $\Delta \delta$ 's may be due to deshielding effects experienced by these protons as the $S$ - and $N$ - donors participate in binding $\mathrm{Ag}^{+}$. Unsurprisingly, lower $\Delta \delta$ values were observed for phenyl protons ('a', 'b' and 'c' in 1a) since they are not in the neighbourhood of the $S$ - and $N$ - donors (Figures 7b). Based on these observations, it was hypothesized that the ligand 1a binds $\mathrm{Ag}^{+}$using its two $S$ - and $\mathrm{N}$ - donor centres to form a tetrahedral complex (Figure 8). 


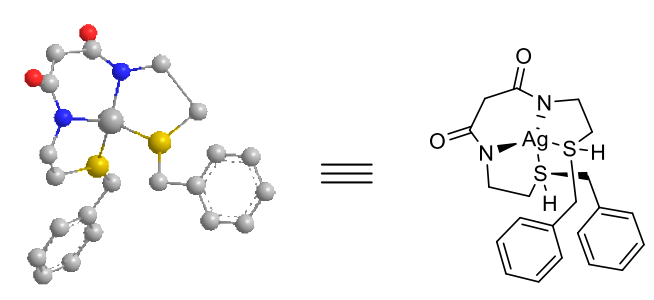

Figure 8. Proposed structure for $[\operatorname{Ag}(\mathbf{1 a})]^{+}$

\section{Conclusion}

A novel room temperature route to access a range of malonamide derivatives 1a and 1e-i in moderate to high yields has been reported. Also, gem diethyl substituted derivatives of 1a (3a-e) were also prepared. Electronic effects at the 4-postion of the aromatic groups in the malonamides 1e-h had little effect on $\mathrm{Ag}^{+}$extraction efficiency but a great effect on metal selectivity, with the electron rich (4methoxy) malonamide $\mathbf{1 f}$ being the most selective for $\mathrm{Ag}^{+}$over $\mathrm{Cu}^{2+}$ and $\mathrm{Pb}^{2+}$. Steric hindrance near the sulphur atom has a small negative effect on $\mathrm{Ag}^{+}$extraction efficiency, while hindrance at the central carbon atom lowers selectivity. The binding stoichiometries of the $\mathrm{Ag}^{+}$complexes of the ligand 1a were determined by mass spectrometry, Job's and mole ratio plots (from ${ }^{1} \mathrm{H}$ NMR titrations) and observed to be a 1:1 type, leading to the predication that $\mathbf{1 a}$ binds $\mathrm{Ag}^{+}$in a tetrahedral fashion. We have therefore shown that by simple modification of the electronics and sterics of the N,N'bis(2(benzylthio)ethyl)malonamides, their selectivity for $\mathrm{Ag}^{+}$recovery from aqueous solutions can be improved over 1a.

\section{Acknowledgments}

The authors would like to thank The Commonwealth Scholarship Commission in the United Kingdom and The University of Warwick for funding Abiodun's $\mathrm{PhD}$.

\section{Declaration of interest statement}

The authors declare no conflict of interest.

\section{References}

[1] Sahan M, Kucuker MA, Demirel B, et al. Determination of Metal Content of Waste Mobile Phones and Estimation of Their Recovery Potential in Turkey. Int J Environ Res Public Health; 16. Epub ahead of print 1 March 2019. DOI: 10.3390/ijerph16050887.

[2] Taillades G, Sarradin J. Silver: High performance anode for thin film lithium ion batteries. $J$ Power Sources 2004; 125: 199-205.

[3] Butterman BWC, Hilliard HE. Mineral Commodity Profiles: Silver. Virginia, 2005. Epub 
ahead of print 2005. DOI: https://pubs.usgs.gov/of/2004/1251/2004-1251.pdf.

[4] Kolpakova N, Sabitova Z, Sachkov V, et al. Determination of $\mathrm{Au}(\mathrm{III})$ and $\mathrm{Ag}(\mathrm{I})$ in Carbonaceous Shales and Pyrites by Stripping Voltammetry. Minerals 2019; 9: 78.

[5] Avarmaa K, Klemettinen L, O'Brien $\mathrm{H}$, et al. Urban mining of precious metals via oxidizing copper smelting. Miner Eng 2019; 133: 95-102.

[6] Jańczewski D, Reinhoudt DN, Verboom W, et al. Tripodal (N-alkylated) CMP(O) and malonamide ligands: Synthesis, extraction of metal ions, and potentiometric studies. New J Chem 2007; 31: 109-120.

[7] Patil AB, Pathak PN, Shinde VS, et al. Synthesis and Evaluation of N,N'-dimethyl-N,N'dicyclohexyl-Malonamide (DMDCMA) as an Extractant for Actinides. Sep Sci Technol 2014; 49: 2927-2932.

[8] Manchanda VK, Pathak PN. Amides and diamides as promising extractants in the back end of the nuclear fuel cycle: An overview. Separation and Purification Technology 2004; 35: 85103.

[9] Daubinet A, Kaye PT. Designer ligands. VIII. Thermal and microwave-assisted synthesis of silver(I)-selective ligands. Synth Commun 2002; 32: 3207.

[10] Ghosh S, Tochtrop GP. A new strategy for the synthesis of -benzylmercaptoethylamine derivatives. Tetrahedron Lett 2009; 50: 1723-1726.

[11] Carroll FI, White JD, Wall ME. Organic Sulfur Compounds. I. Synthesis of secMercaptoalkylamine Hydrochlorides1a,b. J Org Chem 1963; 28: 1236-1239.

[12] Daubinet A. Design, synthesis and evaluation of silver-specific ligands. Rhodes University, 2001.

[13] Sole KC, Hiskey JB. Solvent extraction characteristics of thiosubstituted organophosphinic acid extractants. Hydrometallurgy 1992; 30: 345-365.

[14] Toure M, Arrachart G, Duhamet J, et al. Tantalum and Niobium Selective Extraction by AlkylAcetophenone. Metals (Basel) 2018; 8: 654.

[15] Fielding L. Determination of Association Constants (Ka) from Solution NMR data. Tetrahedron 2000; 56: 6151-6170.

[16] Crane RA, Sinnett DE, Cleall PJ, et al. Physicochemical composition of wastes and co-located environmental designations at legacy mine sites in the south west of England and Wales: Implications for their resource potential. Resour Conserv Recycl 2017; 123: 117-134. 
[17] Ocak Ü, Alp H, Gökçe P, et al. The synthesis of new N2,S2 -Macrocyclic schiff base ligands and investigation of their ion extraction capability from aqueous media. Sep Sci Technol 2006; 41: 391-401.

[18] Pearson RG. Hard and soft acids and bases, HSAB, part 1: Fundamental principles. J Chem Educ 1968; 45: 581.

[19] Hiruta Y, Watanabe T, Nakamura E, et al. Steric hindrance effects in tripodal ligands for extraction and back-extraction of $\mathrm{Ag}^{+}$. RSC Adv 2014; 4: 9791-9798.

[20] Renny JS, Tomasevich LL, Tallmadge EH, et al. Method of continuous variations: Applications of job plots to the study of molecular associations in organometallic chemistry. Angew Chemie - Int Ed 2013; 52: 11998-12013. 